\title{
Microbial Adaptation Due to Gastric Bypass Surgery: The Nutritional Impact
}

\author{
Silke Crommen ${ }^{1}$, Alma Mattes ${ }^{1}$ and Marie-Christine Simon ${ }^{2, *(D)}$ \\ 1 Department of Nutrition and Food Sciences, Nutritional Physiology, University of Bonn, \\ 53115 Bonn, Germany \\ 2 Department of Nutrition and Food Sciences, Nutrition and Microbiota, University of Bonn, \\ 53115 Bonn, Germany \\ * Correspondence: marie-christine.simon@uni-bonn.de; Tel.: +49-228-73-36-80
}

Received: 8 April 2020; Accepted: 22 April 2020; Published: 24 April 2020

\begin{abstract}
Bariatric surgery leads to sustained weight loss and the resolution of obesity-related comorbidities. Recent studies have suggested that changes in gut microbiota are associated with the weight loss induced by bariatric surgery. Several studies have observed major changes in the microbial composition following gastric bypass surgery. However, there are inconsistencies between the reported alterations in microbial compositions in different studies. Furthermore, it is well established that diet is an important factor shaping the composition and function of intestinal microbiota. However, most studies on gastric bypass have not assessed the impact of dietary intake on the microbiome composition in general, let alone the impact of restrictive diets prior to bariatric surgery, which are recommended for reducing liver fat content and size. Thus, the relative impact of bariatric surgery on weight loss and gut microbiota remains unclear. Therefore, this review aims to provide a deeper understanding of the current knowledge of the changes in intestinal microbiota induced by bariatric surgery considering pre-surgical nutritional changes.
\end{abstract}

Keywords: bariatric surgery; Roux-en-Y gastric bypass; gastric bypass surgery; gut microbiome; microbiota; diet; obesity; weight loss

\section{Introduction}

The increasing prevalence of obesity, caused by the changing dietary and exercise habits, seems to have reached epidemic proportions worldwide with more than 650 million adults being affected in 2016 [1]. Western diets, defined by a high fat and low fibre intake, sedentary lifestyle and genetics, are common causes of obesity [2]. Recent findings have suggested that gut microbiota play a role in the onset of obesity by contributing to energy homeostasis and fat storage [3-5] (Figure 1). Furthermore, there is evidence that gut microbiota varies in lean and obese individuals $[4,6,7]$. In particular, there is a difference in the intestinal ratio of Bacteroides and Firmicutes between lean and obese individuals with a greater relative abundance of Firmicutes in obese individuals. At present, only bariatric surgery seems to induce sustained weight loss and resolution of obesity-related morbidities, such as type 2 diabetes mellitus (T2DM), non-alcoholic fatty liver disease (NAFLD), hypertension and cardiovascular disease [8-13], relatively attributable to microbial alterations.

Diet is an important factor shaping the composition and function of intestinal microbiota. However, most studies on gastric bypass have not assessed the impact of dietary intake in general. Additionally, the effects of restrictive diets prior to bariatric surgery, which are recommended for reducing liver fat content and size, on the microbiome composition were not investigated in detail. Thus, the relative impact of bariatric surgery on weight loss and gut microbiota remains unclear. Therefore, this review 
aims to provide a deeper understanding of the changes in intestinal microbiota induced by bariatric surgery considering pre-surgical nutritional changes.

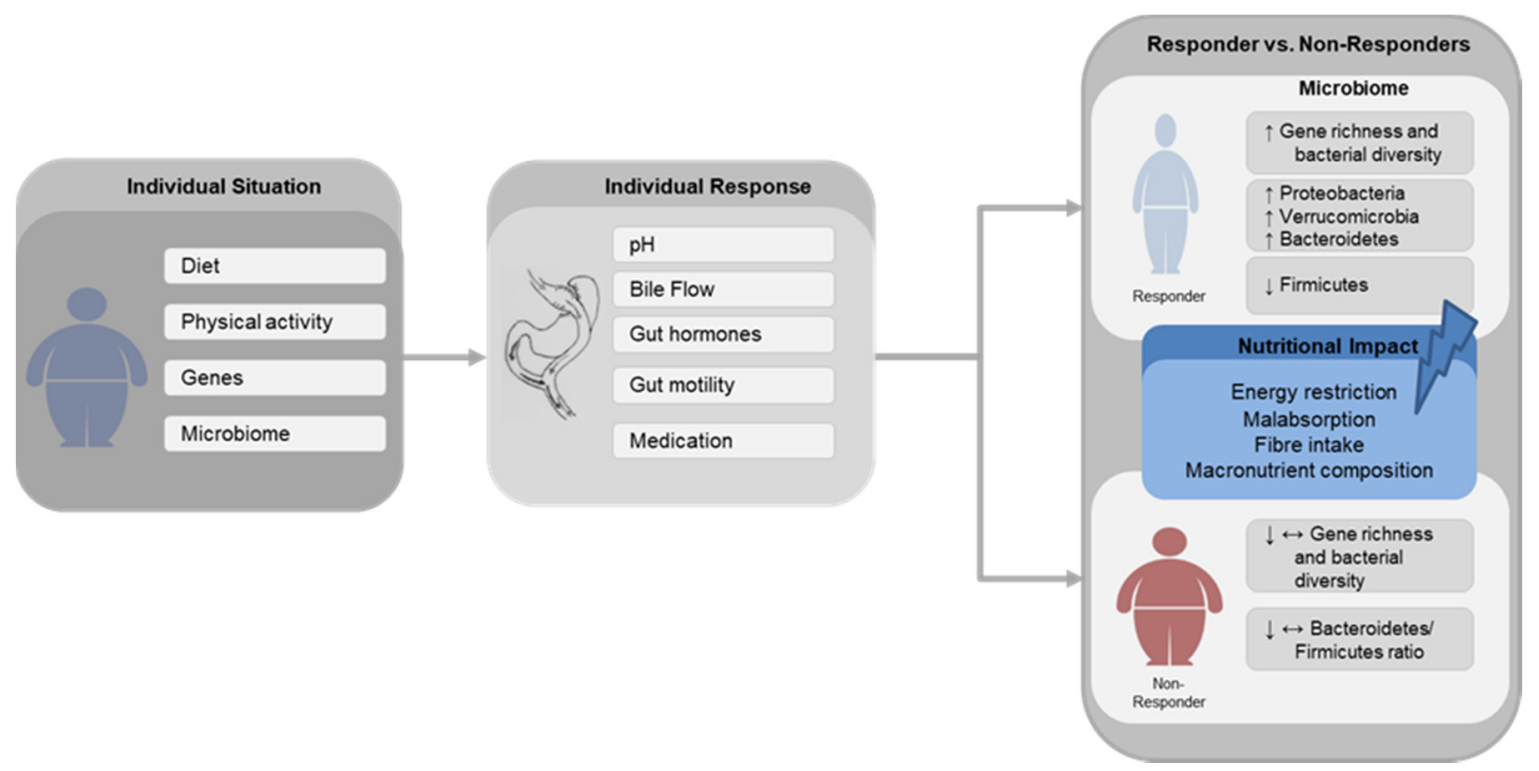

Figure 1. Schematic Illustration Schematic illustration of the main gut microbial changes associated with successful (Responder) and poor (Non-Responder) weight loss after gastric bypass surgery and the possible impact of nutritional factors. Diet, physical activity, genes and gut microbiome composition are widely described factors leading to obesity. Following gastric bypass surgery, the individual response is affected by alterations in $\mathrm{pH}$, bile flow, changes in gut hormones secretion, gut motility and medication usage. $\uparrow$-increase, $\downarrow$-decrease, $\leftrightarrow$ - unchanged.

\section{Materials and Methods}

A systematic literature search was performed on PubMed using the search terms "gastrointestinal microbiome", "gastrointestinal microbiota", "microbiome", "microbiota", "gut microbiome”, "bariatric surgery", "gastric bypass", "Roux-en-Y gastric bypass", RYGB", "mini gastric bypass" and "MGB" individually or in combination. We selected publications between February 2009 and January 2020 containing original research on humans. Of the selected articles, the full texts, as well as the references, were reviewed. If the reference list contained eligible articles, those were also included. All publications not composed in the English language were excluded.

\section{The Intestinal Microbiome in Obesity}

Obesity is associated with changes in the relative abundance of the two dominant bacterial divisions: Bacteroidetes and Firmicutes [4]. Ley et al. discovered in a study comparing lean and obese mice that the ob/ob animals showed a 50\% reduction in the abundance of Bacteroidetes, whereas the level of Firmicutes was higher by a corresponding degree [6]. Analogous differences can be observed in the distal gut microbiota of obese versus lean humans [4]. Animal models have produced evidence for the causal role of intestinal microbiota in the aetiology of obesity and insulin resistance [14]. Turnbaugh et al. showed that faecal microbial transplantation (FMT) of faeces from obese mice into lean, germ-free mice (GF) led to a marked increase in body weight of the recipient animals [4]. The obese phenotype seems to be transmissible and is promoted by microbiota with an increased capacity to harvest energy from the host's diet [4,15]. In line with these findings, a study where faecal microbiota from a pair of twins, discordant in their obesity status, was transplanted into GF mice showed that recipients from the obese donor gained significantly more weight than their counterparts with the lean donor [16]. Such causal relation is scarce for humans; however, one case report depicts a woman successfully treated with FMT who developed new-onset obesity after receiving stool from a healthy but overweight 
donor [17]. Regarding the improvement of obesity-associated metabolic parameters, Zhang et al. found mixed results, as two of the reviewed studies reported an improved peripheral insulin sensitivity after FMT, while some other studies showed no differences in fasting plasma glucose, hepatic insulin sensitivity or BMI after following FMT [18].

The variance of human gut microbiota composition and clinical phenotypes is huge. Based on the Flemish gut flora project and Lifelines DEEP (LLD) cohort, a human core microbiota could be identified. It is expected to be representative of the average gut microbiota composition in the Western European population. While 664 genera were identified, total microbial richness still seems to be underexplored [19]. Age and gender of the study population did not only correlate with microbial composition distance and diversity but also with functional richness [20]. An association between microbiome composition and BMI was small but significant [19]. In the LLD cohort, obese-specific microbial associations were found for lipid compositions in the VLDL and LDL lipoprotein subclasses. In obese individuals, bacterial L-methionine biosynthesis and a Ruminococcus species were associated with cardiovascular phenotypes (i.e., atherosclerosis and liver fat content) [21].

Obese individuals show an altered intestinal ratio of Bacteroidetes and Firmicutes with a greater relative abundance of Firmicutes [15]. A reduction in energy intake is able to lower the ratio because the relative abundance of Bacteroidetes increases as obese individuals lose weight on either a fat- or carbohydrate-restricted low-calorie diet $[4,15]$. Three genera of bacteria are often overrepresented in obese humans, including Bacteroides and Prevotella (both Bacteroidetes) and Ruminococcus (Firmicutes) [22]. In a study by Turnbaugh et al., diet-induced obesity (DIO) produced a bloom in a single uncultured clade within the Mollicutes class of Firmicutes. It became the dominant lineage within distal gut microbiota concurrently accompanied by a division-wide suppression of Bacteroidetes. This finding suggests that the Mollicute lineage has increased fitness relative to other Firmicutes and Bacteroidetes [23].

Bacteria that cause weight gain are thought to induce the expression of genes related to the lipid and carbohydrate metabolism resulting in a greater energy harvest from the diet [24]. Humans get approximately $10 \%$ of their daily energy supply from short-chain fatty acids (SCFAs) produced by the gut bacteria. SCFAs act not only as energy substrates for the host but also as signalling molecules thereby influencing energy intake and metabolism $[25,26]$. However, these SCFA profiles, along with butyrate-producing bacteria, are altered in obese individuals [27].

Using these recent studies, it is not possible to confirm whether associations between obesity and the two dominant bacterial phyla exist because there are discrepancies in the Bacteroidetes/Firmicutes ratio and, therefore, its relation to obesity [24,28]. It is likely that the influence of the gut microbiome on obesity is much more complex than simply an imbalance in the proportion of bacteria phyla [24]. Phylum-wide changes in the gut microbiota cannot be currently considered as biomarkers for obesity [25]. Due to a variety of confounding factors within the human population (heterogeneity in genotype, lifestyle, diet, ethnicity), a suitable definition of an "obese" microbiota is currently impossible [25,29]. In addition, the causal relationship and underlying mechanism remain outstanding [25].

\section{Impact of Gastric Bypass Surgery on Gut Microbiota}

Bariatric surgery is currently the most effective treatment option for achieving sustained weight loss and the resolution of obesity-related comorbidities, such as T2DM, NAFLD, cardiovascular disease and reduced mortality [9-13]. Bariatric surgery is recommended for individuals with a BMI of $\geq 40 \mathrm{~kg} / \mathrm{m}^{2}$ or a BMI of $>35 \mathrm{~kg} / \mathrm{m}^{2}$ with obesity-related comorbidities [30]. There are several bariatric surgery procedures, of which Roux-en-Y gastric bypass (RYGB) is one of the most frequently undertaken procedure globally [31,32], due to the profound weight loss [33] and cardiometabolic improvement observed after this surgery [34,35]. RYGB surgery consists of a reduction of gastric volume by forming a small gastric pouch and a section of jejunum is then connected to the pouch. Thereby, the majority of the stomach, duodenum and the proximal jejunum are excluded from the intestinal tract [36]. 
Bariatric surgery significantly helps to ameliorate biochemical and histologic parameters in patients with NAFLD [37]. Steatosis, Steatohepatitis and liver fibrosis are improved in the majority of patients after surgery [38]. In $84 \%$ of the patients, liver function test values were normalised following bariatric surgery by the end of the first postoperative year. Hereby, both RYGB and SG proved to be similarly effective [39]. RYGB and SG surgery also significantly improved alanine aminotransferase, aspartate aminotransferase, NAFLD activity score and NAFLD fibrosis score [37]. Hepatic insulin resistance was markedly decreased post-surgery, while beta cell function improved due to an increase in postprandial GLP-1 level $[40,41]$. In a study by Feng et al. fasting insulin and 120 min insulin decreased significantly in post-RYGB patients. Decreases in $\mathrm{HbA1c}$ and fasting blood glucose levels were also noticed and reached normal levels at 1-3 months after surgery. Complete NAFLD remission was achieved in $96 \%$ of post-RYGB patients as well as diabetes remission in $48 \%$ of post-RYGB patients, thereby improving cardiovascular risk factors [42].

Generally, initial excess weight loss is about $60 \%-76 \%$ for RYGB patients in the first five years [34,43]. However, the total amount of weight loss shows high inter-individual variability with a large proportion of patients who achieve a large loss of weight (responder) and a subset of patients who fail to achieve the expected weight loss during the first postoperative year or even regain weight afterwards and, therefore, these patients gain little health benefit from the surgery (non-responder) [44-46] (Figure 1). Currently, there are several possible reasons being discussed to account for the high inter-individual variability, such as genetic, epigenetics [47], biological and clinical factors [48-50]. In particular, age, pre-surgical presence of T2DM, higher initial BMI and behavioural problems are associated with poor weight loss after bariatric surgery [51]. Furthermore, it is likely that differential changes in gut microbiota composition also account for the variability seen after gastric bypass procedures. Furet et al. found that a higher ratio of Bacteroides to Prevotella following RYGB leads to higher weight loss and elevated blood leptin levels. However, these associations were dependent on energy intake indicating that the microbial alterations observed after RYGB might be caused by energy restriction [52]. Conversely, in a recent study conducted by Fouladi et al., no differences were found in the gut microbiome composition between patients after RYGB with successful weight loss (SWL) and poor weight loss (PWL). Thus, a humanised mouse model was used to elucidate the possible differences in the composition and function of the microbiome of SWL individuals, PWL individuals and non-surgical control (NSC) individuals who were matched for age and BMI to the SWL-group. Transplantation of faecal samples from SWL, PWL and NSC patients into antibiotic-treated mice revealed that mice colonised with the PWL microbiome gained more weight than mice transplanted with the SWL microbiome even though food intake did not differ between the two groups. In this study, the genus Barnesiella was associated with weight outcome and showed a higher abundance in the PWL recipient mice compared to the SWL and NSC recipient mice. The authors hypothesised that the microbiota from PWL subjects contribute to the observed weight gain after RYGB surgery independent of food intake. This could be due to an increase in energy absorption from diet and increased fat accumulation in adipose tissue inducing low-grade inflammation and metabolic alterations. Rather than compositional differences, there could be at least some functional differences in the gut microbiome of PWL and SWL patients [53].

Several studies have indicated that RYGB surgery not only changed the microbiota composition but also the microbial functions. Thus, enhanced protein degradation, an increase in functional annotations and the associated fatty acid utilisation are widely observed after RYGB surgery [54-57]. This led to the assumption that, after gastric bypass surgery, the energy harvest from the diet is decreased.

Further studies have indicated that the restriction and malabsorption induced by the surgery are not the only cause of the observed metabolic improvements. Rather, findings have suggested that changes in the intestinal microbiota exert considerable influence on surgically induced weight loss and metabolic improvement [55,56,58-61]. Animal studies have shown that RYGB surgery led to a rapid and sustained increase in the abundance of certain microbes, such as Escherichia and Akkermansia, independent of weight loss and energy restriction. After faecal transplantation from RYGB-treated mice to non-operated germ-free mice, recipient mice exhibited a decrease in the rate of weight gain and 
a decreased mass of body fat [62] suggesting that there could be a direct link between the alterations in the gut microbiome and the weight-reducing effects seen after gastric bypass surgery. In a study conducted by Tremaroli et al. RYGB surgery caused long-lasting effects on the composition and functional capacity of the human gut microbiota. Furthermore, the authors colonized germ-free mice with stool from postoperative patients and also demonstrated that the surgically altered microbiota promoted reduced fat deposition in recipient mice. These findings led to the hypothesis that there could be a causal relationship between the microbial alterations following gastric bypass surgery and the observed weight reduction [56].

Several human studies have shown major changes in the gut microbiota composition at the phylum level after gastric bypass (Table 1). Moreover, in terms of the distinct surgical procedures, different microbiota-related alterations have been reported, while most profound changes in gut microbiota were observed after RYGB surgery compared to sleeve gastrectomy (SG) or adjustable gastric banding $[54,59,63,64]$. In general, microbial diversity and richness increased within three months of post-RYGB surgery and continued even after one or two years after the operation $[54,57,60,65]$. Physiological and anatomical modifications induced by RYGB surgery could be responsible for the increase in diversity and microbial richness. This is possibly due to the altered gut environment, particularly an increase in $\mathrm{pH}$ and oxygen content, which allows acid-sensitive bacteria and facultative anaerobic bacteria to colonise [66]. As a result of the higher oxygen level, the relative abundance of the aero-tolerant species E. coli was found to increase after RYGB surgery [52,56,57]. On a taxa level, an increase in Bacteroidetes, Proteobacteria and Verrucomicrobia (e.g., Akkermansia muciniphila) and a decrease in members of the phylum Firmicutes have been consistently observed in humans after gastric bypass procedures compared to the same patients preoperatively or obese patients who have not undergone bariatric surgery (Table 1). Zhang et al. were the first to compare the gut microbiome composition of three normal weight, three morbidly obese and three post-RYGB patients. Lower abundance of Firmicutes, Clostridia and Verrucomicrobia were observed in the RYGB patients compared to those of the normal weight and morbidly obese subjects while the abundance of the class Gammaproteobacteria was markedly increased for the RYGB patients. Additionally, on a family taxonomic level, the relative abundance of Enterobacteriaceae, Fusobacteriaceae and Akkermansia were markedly increased in the post-bariatric group [67]. A recent study comparing the gut microbiota adaptation after RYGB or SG surgery showed different gut microbiota profiles between these surgical procedures [63]. SG surgery was associated with higher levels of Akkermansia, Eubacterium, Haemophilus and Blautia, whereas RYGB surgery resulted in increased levels of Veillonella, Slackia, Granucatiella and Acidaminococcus. Furthermore, among SG subjects an increase in the abundance of Lactobacillales [68,69] and on species level in Bacteroides uniformis [70,71] and Roseburia intestinalis, and a decrease in the phylum Firmicutes [72,73], the family Bifidobacteriaceae [63,73] and the species Coprococcus comes [71,72] and Dorea longicatena [71,72] has been observed. 
Table 1. Changes in the gut microbiota following bariatric surgery in humans.

\begin{tabular}{|c|c|c|c|c|c|c|c|c|c|c|c|}
\hline \multirow{2}{*}{ Reference } & \multirow{2}{*}{ Subjects } & \multirow{2}{*}{$\begin{array}{c}\text { Type of } \\
\text { Surgery } \\
(n)\end{array}$} & \multirow{2}{*}{$\begin{array}{l}\text { Sample } \\
\text { Size }(n)\end{array}$} & \multirow{2}{*}{$\begin{array}{c}\text { Time } \\
\text { Points }\end{array}$} & \multirow{2}{*}{$\begin{array}{l}\text { Pre-BS } \\
\text { Dietary } \\
\text { Intake }\end{array}$} & \multirow{2}{*}{$\begin{array}{l}\text { Post-BS } \\
\text { Dietary } \\
\text { Intake }\end{array}$} & \multirow{2}{*}{$\begin{array}{c}\text { Impact on } \\
\text { Diversity and } \\
\text { Gene Richness }\end{array}$} & \multicolumn{4}{|c|}{ Changes in Relative Abundance } \\
\hline & & & & & & & & Phylum & Class/Order/Family & Genus & Species \\
\hline $\begin{array}{l}\text { Zhang } 2009 \\
\text { [67] }\end{array}$ & $\begin{array}{l}\text { Normal } \\
\text { weight, } \\
\text { obese, } \\
\text { post-BS }\end{array}$ & RYGB & $\begin{array}{l}6 \\
\text { RYGB } 3 \\
\text { NW } 3 \\
\text { MO } 3\end{array}$ & $\begin{array}{l}8-15 \\
\text { mo } \\
\text { post-BS }\end{array}$ & - & - & 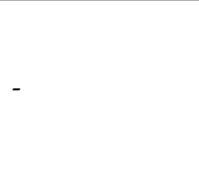 & $\begin{array}{l}\uparrow \text { Verrucomicrobia } \\
\uparrow \text { Fusobacteria } \\
\downarrow \text { Firmicutes }\end{array}$ & $\begin{array}{l}\uparrow \text { Gammaproteobacteria } \\
\uparrow \text { Prevotellaceae } \\
\uparrow \text { Fusobacteriaceae } \\
\uparrow \text { Enterobacteriaceae } \\
\downarrow \text { Clostridia }\end{array}$ & $\begin{array}{l}\downarrow \text { Lachnospira } \\
\uparrow \text { Akkermansia }\end{array}$ & \\
\hline $\begin{array}{l}\text { Furet } 2010 \\
\text { [52] }\end{array}$ & $\begin{array}{l}\text { Post-BS } \\
(7 \text { T2DM), } \\
\text { lean } \\
\text { controls }\end{array}$ & RYGB & $\begin{array}{l}43 \\
\text { RYGB } 30 \\
\text { NW } 13\end{array}$ & $\begin{array}{l}\text { Pre-BS, } \\
3,6 \text { mo } \\
\text { post-BS }\end{array}$ & $\begin{array}{l}\text { 1-h } \\
\text { questioning } \\
\text { period }\end{array}$ & $\begin{array}{l}\text { 1-h } \\
\text { questioning } \\
\text { period }\end{array}$ & - & $\uparrow$ Bacteroidetes & & $\begin{array}{l}\uparrow \text { Bacteroides/ } \\
\text { Prevotella ratio } \\
\downarrow \text { Bifidobacterium } \\
\downarrow \text { Lactobacillus } \\
\downarrow \text { Leuconostoc } \\
\downarrow \text { Pediococcus } \\
\end{array}$ & $\begin{array}{l}\uparrow \text { Escherichia coli } \\
\uparrow \text { Faecalibacterium } \\
\text { prausnitzii }\end{array}$ \\
\hline $\begin{array}{l}\text { Kong } 2013 \\
\text { [65] }\end{array}$ & $\begin{array}{l}\text { Morbidly } \\
\text { obese } \\
\text { women }\end{array}$ & RYGB & 30 & $\begin{array}{l}\text { Pre- BS, } \\
3,6 \text { mo } \\
\text { post-bs }\end{array}$ & $\begin{array}{l}\text { 1-h } \\
\text { questioning } \\
\text { period }\end{array}$ & $\begin{array}{l}\text { 1-h } \\
\text { questioning } \\
\text { period }\end{array}$ & $\uparrow \mathrm{GM}$ richness & $\begin{array}{l}\uparrow \text { Proteobacteria } \\
\downarrow \text { Firmicutes }\end{array}$ & & $\begin{array}{l}\uparrow \text { Alistipes } \\
\uparrow \text { Escherichia } \\
\uparrow \text { Bacteroides } \\
\downarrow \text { Bifidobacterium } \\
\downarrow \text { Lactobacillus } \\
\downarrow \text { Dorea } \\
\downarrow \text { Blautia }\end{array}$ & \\
\hline $\begin{array}{l}\text { Graessler } \\
2013 \text { [55] }\end{array}$ & $\begin{array}{l}\text { Morbidly } \\
\text { obese } \\
\text { subjects }\end{array}$ & RYGB & 6 & $\begin{array}{l}\text { Pre- BS, } \\
3 \text { mo } \\
\text { post-bs }\end{array}$ & - & - & - & $\begin{array}{l}\uparrow \text { Proteobacteria } \\
\uparrow \text { Fusobacteria } \\
\uparrow \text { Verrucomicrobia } \\
\downarrow \text { Bacteroidetes } \\
\downarrow \text { Firmicutes } \\
\downarrow \text { Actinobacteria } \\
\downarrow \text { Cyanobacteria } \\
\uparrow \text { Bacteroidetes/ } \\
\text { Firmicutes ratio }\end{array}$ & & $\begin{array}{l}\uparrow \text { Enterobacter } \\
\uparrow \text { Neurospora } \\
\uparrow \text { Citrobacter } \\
\uparrow \text { Veillonella } \\
\uparrow \text { Salmonella } \\
\downarrow \text { Faecalibacterium } \\
\downarrow \text { Coprococcus } \\
\downarrow \text { Helicobacter } \\
\downarrow \text { Anaerostipes } \\
\downarrow \text { Nakamurella }\end{array}$ & $\begin{array}{l}\uparrow \text { Enterobacter } \\
\text { cancerogenus } \\
\uparrow \text { Veillonella } \\
\text { parvula } \\
\uparrow \text { Veillonella dispar } \\
\uparrow \text { Shigella boydii } \\
\uparrow \text { Salmonella } \\
\text { enerica } \\
\downarrow \text { Lactobacillus } \\
\text { reuteri } \\
\downarrow \text { Treponema } \\
\text { pallidum } \\
\downarrow \text { Mycobacterium } \\
\text { kansasii } \\
\downarrow \text { Faecalibacterium } \\
\text { prausnitzii } \\
\downarrow \text { Clostridium } \\
\text { comes }\end{array}$ \\
\hline
\end{tabular}


Table 1. Cont

\begin{tabular}{|c|c|c|c|c|c|c|c|c|c|c|}
\hline \multirow{2}{*}{ Reference } & \multirow{2}{*}{ Subjects } & \multirow{2}{*}{$\begin{array}{c}\text { Type of } \\
\text { Surgery } \\
(n)\end{array}$} & \multirow{2}{*}{$\begin{array}{l}\text { Sample } \\
\text { Size }(n)\end{array}$} & \multirow{2}{*}{$\begin{array}{l}\text { Time } \\
\text { Points }\end{array}$} & \multirow{2}{*}{$\begin{array}{l}\text { Pre-BS } \\
\text { Dietary } \\
\text { Intake }\end{array}$} & \multirow{2}{*}{$\begin{array}{l}\text { Post-BS } \\
\text { Dietary } \\
\text { Intake }\end{array}$} & \multirow{2}{*}{$\begin{array}{c}\text { Impact on } \\
\text { Diversity and } \\
\text { Gene Richness }\end{array}$} & \multicolumn{3}{|c|}{ Changes in Relative Abundance } \\
\hline & & & & & & & & Phylum & Class/Order/Family & Species \\
\hline $\begin{array}{l}\text { Ward } 2014 \\
\text { [74] }\end{array}$ & $\begin{array}{l}\text { Severely } \\
\text { obese } \\
\text { subjects }\end{array}$ & RYGB & 8 & $\begin{array}{l}\text { Pre- BS, } \\
6 \text { mo } \\
\text { post-bs }\end{array}$ & - & - & (1) & $\begin{array}{l}\text { PPI Users: } \\
\uparrow \text { Bacteroidetes } \\
\uparrow \text { Proteobacteria } \\
\text { PPI non-users: } \\
\downarrow \text { Verrucomicrobia } \\
\downarrow \text { Firmicutes } \\
\downarrow \text { Proteobacteria }\end{array}$ & & \\
\hline $\begin{array}{l}\text { Tremaroli } \\
2015 \text { [56] }\end{array}$ & $\begin{array}{l}\text { Post-BS } \\
\text { women, } \\
\text { non-operate } \\
\text { severely } \\
\text { obese } \\
\text { women }\end{array}$ & $\begin{array}{l}\text { edRYGB } \\
\text { VGB }\end{array}$ & $\begin{array}{l}21 \\
\text { RYGB 7 } \\
\text { VGB 7 } \\
\text { MO } 7\end{array}$ & $\begin{array}{l}9.4 \mathrm{y} \\
\text { post-BS }\end{array}$ & - & - & - & $\begin{array}{l}\uparrow \text { Proteobacteria } \\
\downarrow \text { Firmicutes }\end{array}$ & $\uparrow$ Gammaproteobacteria & $\begin{array}{l}\uparrow \text { Escherichia coli } \\
\downarrow \text { Clostridium } \\
\text { difficile } \\
\downarrow \text { Clostridium } \\
\text { hiranonis } \\
\downarrow \text { Gemella } \\
\text { sanguinis }\end{array}$ \\
\hline $\begin{array}{l}\text { Federico } \\
2016[75]\end{array}$ & $\begin{array}{l}\text { Severely } \\
\text { obese } \\
\text { and } \\
\text { normal } \\
\text { weight }\end{array}$ & BIB & $\begin{array}{l}56 \\
\text { BIB } 28 \\
\text { NW } 28\end{array}$ & $\begin{array}{l}\text { Pre- BS, } \\
6 \text { mo } \\
\text { post-bs }\end{array}$ & $\begin{array}{l}7 \mathrm{~d} \text { food } \\
\text { records }\end{array}$ & $\begin{array}{l}7 \mathrm{~d} \text { food } \\
\text { records }\end{array}$ & - & & & $\begin{array}{l}\uparrow \text { Lactobacillus } \\
\text { crispatus } \\
\uparrow \text { Streptococcus } \\
\text { spp. } \\
\uparrow \text { Megasphaera sp }\end{array}$ \\
\hline $\begin{array}{l}\text { Palleja } 2016 \\
\text { [57] }\end{array}$ & $\begin{array}{l}\text { Morbidly } \\
\text { obese } \\
\text { subjects }\end{array}$ & RYGB & 13 & $\begin{array}{l}\text { Pre- BS, } \\
3,12 \\
\text { mo } \\
\text { post-bs }\end{array}$ & $\begin{array}{l}\text { Weight loss } \\
\text { diet }(8 \% \\
\text { weight } \\
\text { loss })\end{array}$ & - & $\begin{array}{l}\uparrow \text { species } \\
\text { richness } \\
\uparrow \text { gene richness }\end{array}$ & $\begin{array}{l}\uparrow \text { Proteobacteria } \\
\uparrow \text { Fusobacteria }\end{array}$ & & $\begin{array}{l}\text { } \uparrow \text { Escherichia coli } \\
\uparrow \text { Klebsiella } \\
\text { pneumonia } \\
\uparrow \text { Akkermansia } \\
\text { muciniphila } \\
\downarrow \text { Faecalibacterium } \\
\text { prausnitzii } \\
\downarrow \text { Anaerotruncus } \\
\text { colihominis } \\
\downarrow \text { Megasphaeara } \\
\text { micronuciformis } \\
\uparrow \text { Alistipes spp. } \\
\uparrow \text { Streptococcus } \\
\text { spp. } \\
\uparrow \text { Veillonella spp. }\end{array}$ \\
\hline
\end{tabular}


Table 1. Cont.

\begin{tabular}{|c|c|c|c|c|c|c|c|c|c|c|c|}
\hline \multirow{2}{*}{ Reference } & \multirow{2}{*}{ Subjects } & \multirow{2}{*}{$\begin{array}{c}\text { Type of } \\
\text { Surgery } \\
(n)\end{array}$} & \multirow{2}{*}{$\begin{array}{l}\text { Sample } \\
\text { Size }(n)\end{array}$} & \multirow{2}{*}{$\begin{array}{c}\text { Time } \\
\text { Points }\end{array}$} & \multirow{2}{*}{$\begin{array}{l}\text { Pre-BS } \\
\text { Dietary } \\
\text { Intake }\end{array}$} & \multirow{2}{*}{$\begin{array}{l}\text { Post-BS } \\
\text { Dietary } \\
\text { Intake }\end{array}$} & \multirow{2}{*}{$\begin{array}{c}\text { Impact on } \\
\text { Diversity and } \\
\text { Gene Richness }\end{array}$} & \multicolumn{4}{|c|}{ Changes in Relative Abundance } \\
\hline & & & & & & & & Phylum & Class/Order/Family & Genus & Species \\
\hline $\begin{array}{l}\text { Patrone } \\
2016 \text { [58] }\end{array}$ & $\begin{array}{l}\text { Severely } \\
\text { obese }\end{array}$ & BIB & 11 & $\begin{array}{l}\text { Pre- BS, } \\
6 \text { mo } \\
\text { post-bs }\end{array}$ & $\begin{array}{l}\text { Assessment } \\
\text { of dietary } \\
\text { habits }\end{array}$ & $\begin{array}{l}\text { Assessment } \\
\text { of dietary } \\
\text { habits }\end{array}$ & $\begin{array}{l}\downarrow \text { Species } \\
\text { richness }\end{array}$ & & & $\begin{array}{l}\downarrow \text { Lachnospiraceae } \\
\downarrow \text { Clostridiaceae } \\
\downarrow \text { Ruminococca-ceae } \\
\downarrow \text { Eubacteriaceae } \\
\downarrow \text { Coriobacteriaceae } \\
\uparrow \text { Lactobacillus } \\
\uparrow \text { Megasphaera } \\
\uparrow \text { Acidaminococcus }\end{array}$ & \\
\hline $\begin{array}{l}\text { Ilhan } 2017 \\
\text { [54] }\end{array}$ & $\begin{array}{l}\text { Pre-BS } \\
\text { obese, } \\
\text { normal } \\
\text { weight, } \\
\text { post-RYGB } \\
\text { and } \\
\text { post-LAGB }\end{array}$ & $\begin{array}{l}\text { RYGB } \\
\text { LABG }\end{array}$ & $\begin{array}{l}63 \\
\text { RYGB } 24 \\
\text { LAGB } 14 \\
\text { NW } 10 \\
\text { Preb-Ob } \\
15\end{array}$ & $\begin{array}{l}35 \pm 8 \\
\text { mo } \\
\text { post-BS }\end{array}$ & & $\begin{array}{l}4 \mathrm{~d} \text { food } \\
\text { diaries and } \\
\text { FFQ }\end{array}$ & $\uparrow \alpha$-diversity & & $\begin{array}{l}\uparrow \text { Gammaproteobacteria } \\
\uparrow \text { Bacilli } \\
\uparrow \text { Flavobacteria } \\
\uparrow \text { Fusobacteria }\end{array}$ & $\begin{array}{l}\uparrow \text { Escherichia } \\
\uparrow \text { Veillonella } \\
\uparrow \text { Streptococcus } \\
\uparrow \text { Trabulsiella } \\
\uparrow \text { Haemophilus } \\
\uparrow \text { Coprococcus } \\
\uparrow \text { Enterococcus } \\
\downarrow \text { Oscillospira } \\
\downarrow \text { Coprobacillus } \\
\downarrow \text { Bacteroides }\end{array}$ & \\
\hline $\begin{array}{l}\text { Murphy } \\
2017 \text { [59] }\end{array}$ & $\begin{array}{l}\text { Obese } \\
\text { T2DM } \\
\text { subjects }\end{array}$ & $\begin{array}{l}\text { RYGB } \\
\text { SG }\end{array}$ & $\begin{array}{l}14 \\
\text { RYGB } 7 \\
\text { SG } 7\end{array}$ & $\begin{array}{l}1 \mathrm{w} \\
\text { pre-BS, } \\
1 \mathrm{y} \\
\text { post-BS }\end{array}$ & $\begin{array}{l}2 \mathrm{w} \\
\text { Optifast } \\
3 \mathrm{~d} \text { food } \\
\text { diary }\end{array}$ & $\begin{array}{l}3 \mathrm{~d} \text { food } \\
\text { diary }\end{array}$ & $\uparrow \alpha$-diversity & $\begin{array}{l}\uparrow \text { Firmicutes } \\
\uparrow \text { Actinobacteria } \\
\downarrow \text { Bacteroidetes }\end{array}$ & & & \\
\hline $\begin{array}{l}\text { Aron- } \\
\text { Wisnewsky } \\
2018 \text { [60] }\end{array}$ & $\begin{array}{l}\text { Severely } \\
\text { obese } \\
\text { subjects }\end{array}$ & $\begin{array}{l}\text { RYGB } \\
\text { agb }\end{array}$ & $\begin{array}{l}61 \\
\text { RYGB } 41 \\
\text { Agb } 20\end{array}$ & $\begin{array}{l}\text { Pre- BS, } \\
1,3,12 \\
\text { mo } \\
\text { post-bs }\end{array}$ & $\begin{array}{l}\text { Equilibrate } \\
\text { diet }\end{array}$ & - & $\begin{array}{l}\uparrow \text { Microbial } \\
\text { gene richness }\end{array}$ & $\begin{array}{l}\uparrow \text { GU:99 Roseburia } \\
\uparrow \text { GU:225 } \\
\text { Butyricimonas } \\
\text { virosa } \\
\uparrow \text { GU:359 } \\
\text { Butyricimonas } \\
\end{array}$ & & & \\
\hline $\begin{array}{l}\text { Campisciano } \\
2018 \text { [70] }\end{array}$ & $\begin{array}{l}\text { Obese } \\
\text { patients, } \\
\text { normal } \\
\text { weight } \\
\text { controls }\end{array}$ & LGB SG & $\begin{array}{l}40 \\
\text { Sg } 10 \\
\text { LGB } 10 \\
\text { NW } 20\end{array}$ & $\begin{array}{l}\text { Pre- BS, } \\
3 \text { mo } \\
\text { post-bs }\end{array}$ & - & - & $\uparrow \alpha$-diversity & $\begin{array}{l}\uparrow \text { Proteobacteria } \\
\uparrow \text { Firmicutes } \\
\downarrow \text { Actinobacteria } \\
\downarrow \text { Bacteroidetes } \\
\uparrow \text { Firmicutes/ } \\
\text { Bacteroidetes ratio }\end{array}$ & & $\begin{array}{l}\uparrow \text { Prevotella/ } \\
\text { bacteroides ratio } \\
\uparrow \text { Prevotella } \\
\downarrow \text { Bacteroides }\end{array}$ & $\begin{array}{l}\uparrow \text { Bifidobacterium } \\
\text { vulgatus } \\
\uparrow \text { Hafnia alvei } \\
\downarrow \text { Bifidobacterium } \\
\text { uniformis }\end{array}$ \\
\hline
\end{tabular}


Table 1. Cont.

\begin{tabular}{|c|c|c|c|c|c|c|c|c|c|c|c|}
\hline \multirow{2}{*}{ Reference } & \multirow{2}{*}{ Subjects } & \multirow{2}{*}{$\begin{array}{l}\text { Type of } \\
\text { Surgery } \\
(n)\end{array}$} & \multirow{2}{*}{$\begin{array}{l}\text { Sample } \\
\text { Size }(n)\end{array}$} & \multirow{2}{*}{$\begin{array}{l}\text { Time } \\
\text { Points }\end{array}$} & \multirow{2}{*}{$\begin{array}{l}\text { Pre-BS } \\
\text { Dietary } \\
\text { Intake }\end{array}$} & \multirow{2}{*}{$\begin{array}{l}\text { Post-BS } \\
\text { Dietary } \\
\text { Intake }\end{array}$} & \multirow{2}{*}{$\begin{array}{c}\text { Impact on } \\
\text { Diversity and } \\
\text { Gene Richness }\end{array}$} & \multicolumn{4}{|c|}{ Changes in Relative Abundance } \\
\hline & & & & & & & & Phylum & Class/Order/Family & Genus & Species \\
\hline $\begin{array}{l}\text { Cortez } \\
2018[76]\end{array}$ & $\begin{array}{l}\text { Overweight, } \\
\text { class I or } \\
\text { II obesity } \\
\text { T2DM } \\
\text { patients, } \\
\text { medical } \\
\text { care }\end{array}$ & DJB & $\begin{array}{l}21 \\
\text { DJB } 11 \\
\text { SC } 10\end{array}$ & $\begin{array}{l}\text { Pre-BS, } \\
6,12 \\
\text { mo } \\
\text { post-BS }\end{array}$ & - & $\begin{array}{l}\text { SC: diet } \\
\text { formulated } \\
\text { using } \\
\text { total energy } \\
\text { expenditure }\end{array}$ & $\downarrow \alpha$-diversity & $\begin{array}{l}\uparrow \text { Bacteroidetes } \\
\uparrow \text { Verrucomicrobia }\end{array}$ & & $\begin{array}{l}\uparrow \text { Bacteroides } \\
\uparrow \text { Akkermansia } \\
\uparrow \text { Dialister }\end{array}$ & $\begin{array}{l}\uparrow \text { Akkermansia } \\
\text { muciniphila }\end{array}$ \\
\hline $\begin{array}{l}\text { Paganelli } \\
2019[61]\end{array}$ & $\begin{array}{l}\text { Morbidly } \\
\text { obese }\end{array}$ & $\begin{array}{l}\text { RYGB } \\
\text { SG }\end{array}$ & $\begin{array}{l}45 \\
\text { Sg } 22 \\
\text { RYGB } 23\end{array}$ & $\begin{array}{l}\text { Before } \\
\text { VLCD, } \\
2 \mathrm{w} \\
\text { after } \\
\text { VLCD, } \\
1 \mathrm{w}, 3,6 \\
\text { mo } \\
\text { post-bs }\end{array}$ & $\begin{array}{l}2 \mathrm{w} \\
\text { modifast } \\
(500 \mathrm{kcal} / \mathrm{d})\end{array}$ & - & $\begin{array}{l}\text { Post-VLCD: } \\
\downarrow \alpha \text {-diversity } \\
3 \text { and } 6 \text { mo: } \\
\uparrow \alpha \text {-diversity to } \\
\text { baseline level }\end{array}$ & & $\begin{array}{l}\text { Post-VLCD: } \\
\uparrow \text { Rikenellaceae } \\
\downarrow \text { Streptococcaceae } \\
\downarrow \text { Ruminococcaceae } \\
\text { post-BS: } \\
\uparrow \text { Streptococcaceae } \\
\uparrow \text { Enterobacteriaceae } \\
\downarrow \text { Bifidobacteriaceae }\end{array}$ & & \\
\hline $\begin{array}{l}\text { Sanchez- } \\
\text { Alcoholado } \\
2019 \text { [63] }\end{array}$ & $\begin{array}{l}\text { Severely } \\
\text { obese } \\
\text { patients }\end{array}$ & $\begin{array}{l}\text { RYGB } \\
\text { SG }\end{array}$ & $\begin{array}{l}28 \\
\text { RYGB } 14 \\
\text { SG } 14\end{array}$ & $\begin{array}{l}\text { Pre- BS, } \\
3 \text { mo } \\
\text { post-bs }\end{array}$ & - & - & $\sim \alpha$-diversity & $\begin{array}{l}\uparrow \text { Proteobacteria } \\
\uparrow \text { Fusobacteria }\end{array}$ & $\begin{array}{l}\uparrow \text { Fusobacteriaceae } \\
\uparrow \text { Clostridiaceae } \\
\uparrow \text { Enterobacteriaceae } \\
\downarrow \text { Bifidobacteriaceae } \\
\downarrow \text { Peptostrepto- } \\
\text { coccaceae }\end{array}$ & $\begin{array}{l}\downarrow \text { Bifidobacterium } \\
\downarrow \text { Collinsella } \\
\uparrow \text { Slackia } \\
\uparrow \text { Clostridium } \\
\uparrow \text { Veillonella } \\
\uparrow \text { Granucatiella } \\
\uparrow \text { Oscillospira } \\
\uparrow \text { Fusobacterium } \\
\uparrow \text { Granucatiella }\end{array}$ & \\
\hline $\begin{array}{l}\text { Pajecki } \\
2019[77]\end{array}$ & $\begin{array}{l}\text { Super-obese } \\
\text { subjects }\end{array}$ & RYGB & 9 & $\begin{array}{l}\text { Pre- BS, } \\
12,24 \\
\text { mo } \\
\text { post-bs }\end{array}$ & - & - & & $\downarrow$ Proteobacteria & & & \\
\hline $\begin{array}{l}\text { Lee } 2019 \\
{[78]}\end{array}$ & $\begin{array}{l}\text { Mildly or } \\
\text { moderately } \\
\text { obesity } \\
\text { with } \\
\text { T2DM at } \\
10 \% \text { of } \\
\text { weight } \\
\text { loss }\end{array}$ & $\begin{array}{l}\text { RYGB } \\
\text { AGB }\end{array}$ & $\begin{array}{l}12 \\
\text { AGB } 4 \\
\text { RYGB } 4 \\
\text { MWL } 4\end{array}$ & $\begin{array}{l}\text { Pre-BS, } \\
\text { at } 10 \% \\
\text { of } \\
\text { weight } \\
\text { loss, } 9 \\
\text { mo if } \\
10 \% \\
\text { was not } \\
\text { achieved }\end{array}$ & - & - & $\begin{array}{l}\uparrow \alpha \text {-diversity } \\
\uparrow \text { richness }\end{array}$ & $\begin{array}{l}\uparrow \text { Proteobacteria } \\
\uparrow \text { Actinobacteria }\end{array}$ & & $\begin{array}{l}\uparrow \text { Faecalibacterium } \\
\uparrow \text { Akkermansia }\end{array}$ & \\
\hline
\end{tabular}


Table 1. Cont.

\begin{tabular}{|c|c|c|c|c|c|c|c|c|c|c|c|}
\hline \multirow{2}{*}{ Reference } & \multirow{2}{*}{ Subjects } & \multirow{2}{*}{$\begin{array}{c}\text { Type of } \\
\text { Surgery } \\
(n)\end{array}$} & \multirow{2}{*}{$\begin{array}{l}\text { Sample } \\
\text { Size }(n)\end{array}$} & \multirow{2}{*}{$\begin{array}{c}\text { Time } \\
\text { Points }\end{array}$} & \multirow{2}{*}{$\begin{array}{l}\text { Pre-BS } \\
\text { Dietary } \\
\text { Intake }\end{array}$} & \multirow{2}{*}{$\begin{array}{l}\text { Post-BS } \\
\text { Dietary } \\
\text { Intake }\end{array}$} & \multirow{2}{*}{$\begin{array}{c}\text { Impact on } \\
\text { Diversity and } \\
\text { Gene Richness }\end{array}$} & \multicolumn{4}{|c|}{ Changes in Relative Abundance } \\
\hline & & & & & & & & Phylum & Class/Order/Family & Genus & Species \\
\hline $\begin{array}{l}\text { Fouladi } \\
2019[53]\end{array}$ & $\begin{array}{l}\text { Post-RYGB } \\
\text { with } \\
\text { successful } \\
\text { or poor } \\
\text { weight } \\
\text { loss, } \\
\text { non-surgical } \\
\text { controls }\end{array}$ & $\begin{array}{l}\text { RYGB } \\
1\end{array}$ & $\begin{array}{l}18 \\
\text { SWL } 6 \\
\text { PWL } 6 \\
\text { NSC } 6\end{array}$ & $\begin{array}{l}2-5 \\
\text { years } \\
\text { post-BS }\end{array}$ & - & $\begin{array}{l}\text { 24-h recall } \\
\text { for } 3 \text { days }\end{array}$ & $\begin{array}{l}\uparrow \alpha \text {-diversity } \\
\uparrow \text { richness } \\
\text { PWL vs. NW } \\
\uparrow \text { diversity }\end{array}$ & & $\begin{array}{l}\uparrow \text { Micrococcales } \\
\uparrow \text { Lactobacillales }\end{array}$ & $\begin{array}{l}\uparrow \text { Rothia } \\
\uparrow \text { Streptococcus } \\
\text { PWL vs. NW: } \\
\uparrow \text { Oscullibacter } \\
\uparrow \text { Lactobacillus } \\
\uparrow \text { Enterobacter } \\
\uparrow \text { Akkermansia }\end{array}$ & \\
\hline $\begin{array}{l}\text { Gutierrez- } \\
\text { Repiso } \\
2019 \text { [44] }\end{array}$ & $\begin{array}{l}\text { Post-RYGB } \\
\text { with } \\
\text { primary } \\
\text { failure, } \\
\text { weight } \\
\text { regain or } \\
\text { successful } \\
\text { weight } \\
\text { loss }\end{array}$ & RYGB & $\begin{array}{l}24 \\
\text { SWL } 6 \\
\text { Primary } \\
\text { failure } 6 \\
\text { Weight } \\
\text { regain } 12\end{array}$ & $\begin{array}{l}8.3 \pm \\
1.7 \\
\text { years } \\
\text { post-BS }\end{array}$ & - & - & $\sim \alpha$-diversity & & & $\begin{array}{l}\text { Success vs. weight } \\
\text { regain: } \\
\uparrow \text { Butyrivibrio } \\
\uparrow \text { Lachnospira } \\
\uparrow 5-7 \text { N15 } \\
\uparrow \text { Sacina } \\
\uparrow \text { Alkaliphilus } \\
\uparrow \text { Pseudo- } \\
\text { altermonas } \\
\uparrow \text { Cetobacterium } \\
\uparrow \text { AF12 }\end{array}$ & \\
\hline $\begin{array}{l}\text { Palmisano } \\
2019 \text { [64] }\end{array}$ & $\begin{array}{l}\text { Obese } \\
\text { patients, } \\
\text { normal } \\
\text { weight } \\
\text { controls }\end{array}$ & $\begin{array}{l}\text { RYGB } \\
\text { SG }\end{array}$ & $\begin{array}{l}50 \\
\text { RYGB 9 } \\
\text { Sg } 16 \\
\text { NW } 25\end{array}$ & $\begin{array}{l}\text { Pre- BS, } \\
3,6 \text { mo } \\
\text { post-bs }\end{array}$ & $\begin{array}{l}\text { Food } \\
\text { preferences }\end{array}$ & $\begin{array}{l}\text { Food } \\
\text { preferences }\end{array}$ & $\sim \alpha$-diversity & $\begin{array}{l}\uparrow \text { Proteobacteria } \\
\uparrow \text { Fusobacteria } \\
\uparrow \text { Verrucomicrobia } \\
\downarrow \text { Bacteroidetes } \\
\downarrow \text { Firmicutes }\end{array}$ & $\uparrow$ Gammaproteobacteria & & $\begin{array}{l}6 \text { mo: } \\
\uparrow \text { Akkermansia } \\
\text { muciniphila } \\
\downarrow \text { Veillonella } \\
\text { atypical } \\
\downarrow \text { Veillonella dispar } \\
\downarrow \text { Streptococcus } \\
\text { gordonii } \\
\downarrow \text { Streptococcus } \\
\text { australis } \\
\uparrow \text { Yokenella } \\
\text { regensburgei } \\
\uparrow \text { Fusobacterium } \\
\text { varium }\end{array}$ \\
\hline $\begin{array}{l}\text { Shen } 2019 \\
\text { [79] }\end{array}$ & $\begin{array}{l}\text { Severely } \\
\text { obese } \\
\text { with and } \\
\text { without } \\
\text { T2DM }\end{array}$ & $\begin{array}{l}\text { RYGB } \\
\text { SG }\end{array}$ & $\begin{array}{l}26 \\
\text { RYGB } 19 \\
\text { SG } 7\end{array}$ & $\begin{array}{l}\text { Pre-BS, } \\
3,6,12 \\
\text { mo } \\
\text { post-BS }\end{array}$ & - & - & $\begin{array}{l}6 \text { mo: } \\
\uparrow \text { for } \\
\alpha \text {-diversity } \\
\uparrow \beta \text {-diversity } \\
12 \text { mo: } \\
\text { Tend to pre-BS } \\
\text { levels }\end{array}$ & $\begin{array}{l}3 \text { and } 6 \text { mo: } \\
\uparrow \text { Verrucomicrobia } \\
\uparrow \text { Proteobacteria } \\
12 \text { mo: } \\
\text { trend diminished }\end{array}$ & & $\uparrow$ Akkermansia & \\
\hline
\end{tabular}


Table 1. Cont

\begin{tabular}{|c|c|c|c|c|c|c|c|c|c|c|c|}
\hline \multirow{2}{*}{ Reference } & \multirow{2}{*}{ Subjects } & \multirow{2}{*}{$\begin{array}{c}\text { Type of } \\
\text { Surgery } \\
(n)\end{array}$} & \multirow{2}{*}{$\begin{array}{l}\text { Sample } \\
\text { Size }(n)\end{array}$} & \multirow{2}{*}{$\begin{array}{c}\text { Time } \\
\text { Points }\end{array}$} & \multirow{2}{*}{$\begin{array}{l}\text { Pre-BS } \\
\text { Dietary } \\
\text { Intake }\end{array}$} & \multirow{2}{*}{$\begin{array}{l}\text { Post-BS } \\
\text { Dietary } \\
\text { Intake }\end{array}$} & \multirow{2}{*}{$\begin{array}{c}\text { Impact on } \\
\text { Diversity and } \\
\text { Gene Richness }\end{array}$} & \multicolumn{4}{|c|}{ Changes in Relative Abundance } \\
\hline & & & & & & & & Phylum & Class/Order/Family & Genus & Species \\
\hline $\begin{array}{l}\text { Al Assal } \\
2020[80]\end{array}$ & $\begin{array}{l}\text { Obese } \\
\text { T2DM } \\
\text { women }\end{array}$ & RYGB & 24 & $\begin{array}{l}\text { Pre-BS, } \\
3,12 \\
\text { mo } \\
\text { post-BS }\end{array}$ & $\begin{array}{l}7 \mathrm{~d} \text { records } \\
(1700 \\
\mathrm{kcal} / \mathrm{d})\end{array}$ & $7 \mathrm{~d}$ records & $\uparrow \mathrm{GM}$ richness & $\begin{array}{l}3 \text { mo: } \\
\uparrow \text { Proteobacteria } \\
\uparrow \text { Firmicutes } \\
\uparrow \text { Actinobacteria } \\
12 \text { mo: } \\
\downarrow \text { Firmicutes/ } \\
\text { bacteroidetes ratio }\end{array}$ & & $\begin{array}{l}3 \text { mo: } \\
\uparrow \text { Veillonella } \\
\uparrow \text { Streptococcus } \\
\uparrow \text { Gemella } \\
\uparrow \text { Oribacterium } \\
\uparrow \text { Atopobium } \\
\uparrow \text { one unclassified } \\
\text { Lactobacillus } \\
\text { genus } \\
\uparrow \text { Leptotrichia } \\
\uparrow \text { Neisseria } \\
\uparrow \text { one unclassified } \\
\text { Pasteurellaceae } \\
\text { genus } \\
\downarrow \text { Faecalibacterium }\end{array}$ & \\
\hline
\end{tabular}

$\uparrow —$ increase, $\downarrow$-decrease, $\sim$ - unchanged, $\uparrow$ tended to increase, AGB—adjustable gastric banding, BIB—biliointestinal bypass, BS—-bariatric surgery, DJB-duodenal-jejunal bypass,
FFQ—food frequency questionnaire, GM—gut microbial richness, LAGB—-laparoscopic adjustable gastric banding, LGB—laparoscopic gastric bypass, MO-morbidly obese, MWL—-medical weight loss, NSC—non-surgical control, PPI—proton-pump inhibitor, PWL—poor weight loss, RYGB—Roux-en-Y gastric bypass, SC—standard care group, SG—sleeve gastrectomy, SWL—successful weight loss, T2DM—type 2 diabetes mellitus, VGB—vertical banded gastroplasty, VLCD—very-low-calorie diet. 
Some taxonomic changes following RYGB surgery have been associated with the resolution of comorbidities and weight loss. High levels of Gammaproteobacteria phylotypes and Lactobacillus have been associated with weight loss after RYGB surgery [65]. Furthermore, a negative correlation between blood glucose levels and Lactobacillus abundance has been found. This association remained significant even after correction for calorie intake [58]. An increase in F. prausnitzii has been observed after RYGB surgery and was shown to be inversely correlated with inflammatory markers [52]. However, this correlation was dependent on calorie intake. In this study, the microbial adaptation following RYGB surgery could be due to energy restriction. On the contrary, Graessler et al. observed decreased levels of F. prausnitzii, which were correlated with a reduction in C-reactive protein [55].

However, the microbial changes observed following gastric bypass are not consistent between studies (Table 1). This could be caused by differences in the techniques used for microbiome analysis (e.g., pyrosequencing, metagenomic, RT-qPCR), different time frames of follow up post-surgery, pre-surgical presence of T2DM and medication (e.g., proton-pump inhibitors, statins or antidiabetic drugs) used in the cohorts. One of the weak points in some studies is that the comparison of gut microbiota pre- and post-surgery is missing (Table 1). Considering the inter-individual variation in the gut microbiota composition, using obese subjects as controls does not entirely represent preoperative microbiota. Furthermore, results could be biased by differences in food intake, reduced digestion and changes in food choices and preferences. It is well documented that diet is an important factor shaping the composition and function of intestinal microbiota. Following gastric bypass surgery, dietary intake is altered both in quantity and quality. Bariatric surgery leads to a $40 \%-50 \%$ reduction in energy intake in the first six months post-surgery. Compared to pre-surgery values, energy intake is decreased by $1215 \mathrm{kcal} / \mathrm{d}$ after RYGB surgery [81]. Studies have indicated an improvement in food intake with a reduced fat and carbohydrate intake in the early postoperative period [82]. After the first year, food intake tended to return to pre-surgery habits [82,83].

In many bariatric surgical centres, a very low-calorie diet (VLCD) is regularly prescribed before surgery. Excess body fat is known to exacerbate the technical aspects of surgery increasing both the operating time and the risk of complications [28]. Thus, it could be assumed that at the time of baseline faecal sampling, the majority of patients already had been on a VLCD resulting in atypical microbial compositions and, therefore, an incorrect baseline. To the best of our knowledge, there is only one study considering restrictive diets prior to bariatric surgery [61]. Moreover, several of the studies we reviewed did not assess food intake post-surgery or adjust for changes in food intake and digestion after data collection. Thus, whether the observed alterations in gut microbiota are a direct consequence of surgery itself or of the alterations in food intake and/or weight loss still remains unclear. For this purpose, mouse models, using calorie restriction or sham-operated pair-fed individuals as diet-matched controls could be a helpful tool. There is some evidence from experimental studies on rats, which indicates that the observed effects on gut microbiota composition and diversity after RYGB surgery are associated with surgery itself $[84,85]$. Guo et al. found similar glucose improvement and increased gut microbiota diversity 10 weeks after RYGB and SG surgery compared with the sham-operated groups (pair-fed as RYGB or fed ad litidum). The presence of the pair-fed group as an RYGB diet-matched control group indicated that the increased diversity is caused by RYGB surgery and consequently may be independent of food intake. Following RYGB surgery a higher relative abundance of Proteobacteria/Gammaproteobacteria and Betaproteobacteria and an increase in levels of Fusobacteria and Clostridium compared to SG and sham-operated groups was observed, while SG caused an increase in the relative abundance of Actinobacteria compared with the other groups. Most of the 12 discriminate microbial genera that were affected by RYGB surgery, correlated with alterations in metabolic phenotype. However, only $28.6 \%$ of these correlations remained significant after adjustment for body weight and four discriminant genera negatively correlated with serum insulin level independent of food intake and weight loss after RYGB surgery [84].

In addition to the microbial changes after bariatric surgery, Paganelli et al. also investigated short-term alterations in the microbiome following a VLCD prior to either RYGB or SG surgery. 
They found that such a crash diet $(500 \mathrm{kcal} / \mathrm{d})$ induced profound but temporary changes in the gut microbiome diversity and composition. Contrary to the crash diet, surgery was associated with the early and persistent replacement of distinct bacterial taxa and restoration of the gut microbial diversity. The VLCD resulted in a temporal increase in the relative abundance of Bifidobacterium and a decrease in the relative abundance of Streptococcaceae, whereas the opposite effect was observed after surgery. These alterations persisted for at least six months. With regard to relative abundance and beta-diversity of enteric bacteria, there were no differences between patients undergoing either RYGB or SG surgery: neither prior to surgery nor at any time point afterwards. In addition, the weight loss in patients was comparable between the two surgeries. Hence, the authors concluded that the comparable weight loss could be causative for the similar changes in gut microbiota composition observed after both surgery types. In this study, bariatric surgery itself, unlike a VLCD, resulted in marked and sustained alterations of the gut microbiota composition [61]. Murphy et al. examined enteric microbial changes after RYGB or SG surgery in obese patients with T2DM. At the time of baseline faecal sampling, all patients had been on a VLCD using a formula diet (Optifast, 152-207 kcal per serving) plus green, non-starchy vegetables daily for at least two weeks. RYGB surgery resulted in an increase in the gut microbiota diversity, which was not observed after SG. Three major phyla changes with increased Firmicutes and Actinobacteria and decreased Bacteroidetes phyla were detected after RYGB surgery. Contrarily, SG surgery caused an increase in Bacteroidetes phyla. An increase in Roseburia species was associated with diabetes remission for both types of surgery [59].

Given the known association between food intake and gut microbial richness and the dramatic changes in food intake after gastric bypass surgery, $\mathrm{Al}$ Assal et al. recently investigated the microbial profile from obese diabetic women before and after RYGB surgery while taking into account the nutritional impact. Therefore, the authors evaluated the microbiome from 25 patients before, three months after and 12 months after surgery. Food intake was calculated using a seven-day food record. RYGB surgery resulted in changes in the relative abundance of some gut bacteria genera (Table 1), increased gut microbial richness and induced a decrease in the Firmicutes to Bacteroidetes ratio 12 months post-RYGB surgery. Microbial richness level was correlated with diet composition both in the pre- and post-surgery state. However, increased microbial richness was not associated with total diabetes remission. The genus richness had a positive correlation with the fibre/lipid intake ratio and the fibre intake alone and it also had a negative correlation with the lipid intake both pre and postoperatively. Specifically, a direct correlation was exhibited between the lipid intake and levels of one unclassified genus of Acidaminococcaceae and an inverse correlation was exhibited between the lipid intake and Parabacteroides levels. Protein intake had an inverse correlation with Akkermansia levels and a direct correlation with levels of one unclassified Veillonellaceae. Interestingly, only patients achieving diabetes remission increased their total soluble and insoluble fibre intake and decreased saturated fat intake [80]. Thus, additionally to bariatric surgery-induced microbial adaptation, the diet itself, on a macronutrient level, affects the microbial composition and consequently weight loss.

\section{Diet and Microbiome}

As previously stated, diet plays a key role in shaping the microbiome. Alterations in dietary habits can induce microbial shifts within $24 \mathrm{~h}$, which are determined by the competition for substrates and the toleration of gut conditions $[15,86]$. The strongest dietary influence on the formation of the microbial metabolites is exerted by non-digestible carbohydrates, protein and fat [86]. In studies with digestible carbohydrates, where humans were fed high levels of glucose, fructose and sucrose, an increased relative abundance of Bifidobacteria and a reduced count of Bacteroides was observed. Non-digestible carbohydrates, however, seem to have a positive effect on microbiota gene richness. A diet rich in non-digestible carbohydrates increases the abundance of intestinal Bifidobacteria and lactic acid bacteria [15]. Bifidobacteria are beneficial bacteria in the gut whose growth can be stimulated with prebiotic fibre ingestion [87]. Prebiotics, which are defined as non-digestible dietary components that benefit their host's health via the selective stimulation of growth and/ or activity of certain 
microorganisms, induce shifts in the gut microbiome [15]. Major products of the fermentation of carbohydrates, such as the short-chain fatty acids propionate and butyrate, serve as an energy source for the host and provide anti-inflammatory and anti-apoptotic effects [86]. The Prevotella enterotype, which is one of the leading causes of inter-individual gut microbiota variations, is associated with high values of carbohydrates and simple sugars in the diet. It is hypothesised to be sensitive to long-term fibre intake $[88,89]$. Vegetarians and members of agrarian societies show enrichment in Prevotella $[88,90]$.

Protein consumption positively correlates with an overall microbial diversity. Mainly the counts of bile-tolerant anaerobes, such as Bacteroides, Alistipes and Bilophila, increase after the consumption of animal-based protein $[15,91]$. At the same time some bacteria that are generally regarded as a beneficial decrease in their abundance on a high protein diet, including the butyrate producer F. prausnitzii, Ruminococcus and the mucin degrading Akkermansia [91]. The effect also depends on the source of protein, for example, an increased dietary protein content through the consumption of red meat is associated with an increased formation of toxic bacterial metabolites that create a less favourable gut environment [86]. Plant-derived proteins seem to be more favourable for promoting beneficial microbiota that have positive effects on the host's metabolism [91], which could be related to the protein quality itself or the indirect increase of fibre as a side effect of the consumption of plant-derived proteins. Whereas an animal-based diet not only increases the bile-tolerant microorganisms but at the same time reduces the abundance of Firmicutes, such as Roseburia, Eubacterium rectale and Ruminococcus bromii, which preferentially metabolise dietary plant polysaccharides [89]. As observed for an animal-based protein diet, a high-fat diet also increases the total anaerobic microflora as well as the abundance of Bacteroides [15]. A changed microbiota induced by a high-fat diet can even trigger metabolic inflammation through a greater gut permeability allowing Lipopolysaccharides to enter systemic circulation [92].

The Western diet, which as a dietary pattern is typically high in animal protein, sugar, starch, fat and low in fibre, combines these different aspects discussed above and leads to a distinct decrease in numbers of both total bacteria and beneficial Bifidobacterium and Eubacterium species [14,90]. The cecal community of individuals consuming Western-associated diets contain a higher relative abundance of Firmicutes and a lower relative abundance of Bacteroidetes [23]. This was highlighted in a study by De Filippo et al. which compared the gut microbiome of children from Europe (Florence, Italy) and rural Africa (Burkina Faso). The diet from children in Burkina Faso was low in fat and animal protein but rich in starch, fibre and plant polysaccharides and, therefore, resulted in a significant enrichment in Bacteroidetes and a depletion in Firmicutes. A unique abundance of Prevotella and Xylanibacter was found. This allows for the supposition that the exposure to a large variety of environmental microbes, which is associated with the high-fibre diet of agrarian societies, could have a positive effect on potentially beneficial bacterial genomes. The European children, on the other hand, consumed a typical Western diet. They showed an increased Firmicutes/Bacteroidetes ratio probably induced by the high-calorie diet. However, total microbial richness and biodiversity was lower in the European as opposed to the African children [90].

In addition to the studies on high-caloric diets, there are also studies about VLCD and their influence on the gut microbiome. Louis et al. managed to detect some variations at the genera and species level but none at the phyla level after consumption of the VLCD in the form of formula (OPTIFAST ${ }^{\circledR}$ Nestlé, $800 \mathrm{kcal} / \mathrm{d}$ ) over a period of three months. The formula was enriched with inulin to improve bowel movement. Despite the supplementation with inulin, butyrate-producing Roseburia decreased during the phase of the intervention. Other than the response of the microbiota to the dietetic and lifestyle intervention, there were no significant changes observed and the initial gut microbiome was restored at a taxonomical and functional level one year after the very low-calorie diet. The only exception was an increase in the abundance of Akkermansia which persisted throughout the study [93]. Several other studies showed similar outcomes when using the same VLCD formula. These other studies also reported alterations in the gut microbial diversity and bacterial metabolism 
during the three months of intervention phase but the VLCD-induced changes diminished after ending the intervention and went back to baseline levels during the weight maintenance phase [94]. Furthermore, the energy restriction did not affect alpha-diversity and did not trigger consistent shifts in the phylogenetic composition between individuals [95]. Only one study by Damms-Machado et al. demonstrated a change in the Bacteroidetes/Firmicutes ratio after 12 weeks of a VLCD programme. The dietary intervention resulted in a reduced abundance of Bacteroidetes in favour of Firmicutes. This rise is mainly due to butyrate-producing bacterial strains that can process the inulin-enriched formula. Despite the overall growth in Firmicutes, which is typically associated with an increased energy harvest for the host, the specific growth of butyrate-producing species may result in positive metabolic effects [72].

In addition to its influence on microbial composition, preoperative weight loss with a VLCD can help simplify gastric surgeries [29]. In particular, an enlarged liver compromises the visibility of the gastroesophageal area making it more friable and, therefore, more prone to bleeding [26]. In a study by Lewis et al., after six weeks of an Optifast ${ }^{\circledR}$ diet, the mean liver volume was reduced by $14.7 \%$ and the mean liver fat by $43 \%$. This suggests that the reduction in liver volume is due to a loss of fat [96]. Similar results can be found in a clinical trial by Gils Contreras et al. where the liver volume was reduced by $15.6 \%$ after three weeks of VLCD. The reduction was directly related to the baseline BMI [26]. Generally, the highest decrease in liver volume seems to be achieved in the first two weeks of the diet $[28,29]$. Although a reduced liver size might positively influence the surgeon's perceived difficulty of the procedure, there were no differences found regarding mean operating time, estimated blood loss or intraoperative complications compared to control groups without preoperative VLCD $[28,97]$. Only the risk of postoperative complications, especially infections, decreased after the preoperative diet in comparison to no prior diet [28]. However, most studies do not assess the diet-induced microbial alterations prior to surgery resulting in a biased comparison of the atypical microbial compositions at baseline and after surgery.

\section{Conclusions}

Even though bariatric surgery is established as the only effective treatment option for achieving sustained weight loss and metabolic improvement, the exact mode of action is still unresolved. The total extent of weight loss exhibits high inter-individual variation in patients (responder vs non-responders). Our research shows that it is likely that differential changes in gut microbiota account for the variability found after gastric bypass surgeries. In addition to the surgically induced microbial adaptation, the diet itself, on a macronutrient level, affects the microbial composition and, therefore, weight loss. It is well documented that diet is an important factor shaping the composition and function of intestinal microbiota. However, most studies on bariatric surgery do not assess food intake pre- or post-surgery or adjust for changes in food intake and digestion afterwards. Thus, further research efforts are needed to deepen the understanding of gut microbial changes after bariatric surgery, which induce weight loss and metabolic improvements, and the relative impact of nutrition.

Author Contributions: S.C., A.M. and M.-C.S. wrote the paper; M.-C.S. conceived and supervised the project. All authors have read and agreed to the published version of the manuscript.

Funding: This work was funded by the Department of Nutrition and Food Sciences, Nutrition and Microbiota, University of Bonn and supported by the grant no. 01EA1707 of the German Federal Ministry of Education and Research.

Acknowledgments: The authors would like to thank Katherine Macmillan for the professional English editing service.

Conflicts of Interest: The authors declare no conflicts of interest. 


\section{References}

1. World Health Organization. WHO|Obesity. Available online: https://www.who.int/topics/obesity/en/ (accessed on 21 March 2020).

2. Blüher, M. Obesity: Global epidemiology and pathogenesis. Nat. Rev. Endocrinol. 2019, 15, $288-298$. [CrossRef] [PubMed]

3. Cani, P.D.; Delzenne, N.M. The role of the gut microbiota in energy metabolism and metabolic disease. Curr. Pharm. Des. 2009, 15, 1546-1558. [CrossRef] [PubMed]

4. Turnbaugh, P.J.; Ley, R.E.; Mahowald, M.A.; Magrini, V.; Mardis, E.R.; Gordon, J.I. An obesity-associated gut microbiome with increased capacity for energy harvest. Nature 2006, 444, 1027-1031. [CrossRef] [PubMed]

5. Bäckhed, F.; Ding, H.; Wang, T.; Hooper, L.V.; Koh, G.Y.; Nagy, A.; Semenkovich, C.F.; Gordon, J.I. The gut microbiota as an environmental factor that regulates fat storage. Proc. Natl. Acad. Sci. USA 2004, 101, 15718-15723. [CrossRef] [PubMed]

6. Ley, R.E.; Bäckhed, F.; Turnbaugh, P.; Lozupone, C.A.; Knight, R.D.; Gordon, J.I. Obesity alters gut microbial ecology. Proc. Natl. Acad. Sci. USA 2005, 102, 11070-11075. [CrossRef]

7. Zuo, H.-J.; Xie, Z.-M.; Zhang, W.-W.; Li, Y.-R.; Wang, W.; Ding, X.-B.; Pei, X.-F. Gut bacteria alteration in obese people and its relationship with gene polymorphism. World J. Gastroenterol. 2011, 17, 1076-1081. [CrossRef]

8. Oliveira, S.C.; Neves, J.S.; Souteiro, P.; Pedro, J.; Magalhães, D.; Guerreiro, V.; Bettencourt-Silva, R.; Costa, M.M.; Varela, A.; Barroso, I.; et al. Impact of Bariatric Surgery on Long-term Cardiovascular Risk: Comparative Effectiveness of Different Surgical Procedures. Obes. Surg. 2020, 30, 673-680. [CrossRef]

9. Courcoulas, A.P.; Gallagher, J.W.; Neiberg, R.H.; Eagleton, E.B.; DeLany, J.P.; Lang, W.; Punchai, S.; Gourash, W.; Jakicic, J.M. Bariatric Surgery vs Lifestyle Intervention for Diabetes Treatment: 5-Year Outcomes From a Randomized Trial. J. Clin. Endocrinol. Metab. 2020, 105. [CrossRef]

10. Singh, P.; Subramanian, A.; Adderley, N.; Gokhale, K.; Singhal, R.; Bellary, S.; Nirantharakumar, K.; Tahrani, A.A. Impact of bariatric surgery on cardiovascular outcomes and mortality: A population-based cohort study. Br. J. Surg. 2020, 107, 432-442. [CrossRef]

11. Khorgami, Z.; Shoar, S.; Saber, A.A.; Howard, C.A.; Danaei, G.; Sclabas, G.M. Outcomes of Bariatric Surgery Versus Medical Management for Type 2 Diabetes Mellitus: A Meta-Analysis of Randomized Controlled Trials. Obes. Surg. 2019, 29, 964-974. [CrossRef]

12. Schauer, P.R.; Bhatt, D.L.; Kirwan, J.P.; Wolski, K.; Aminian, A.; Brethauer, S.A.; Navaneethan, S.D.; Singh, R.P.; Pothier, C.E.; Nissen, S.E.; et al. Bariatric Surgery versus Intensive Medical Therapy for Diabetes-5-Year Outcomes. N. Engl. J. Med. 2017, 376, 641-651. [CrossRef] [PubMed]

13. Buchwald, H.; Estok, R.; Fahrbach, K.; Banel, D.; Jensen, M.D.; Pories, W.J.; Bantle, J.P.; Sledge, I. Weight and type 2 diabetes after bariatric surgery: Systematic review and meta-analysis. Am. J. Med. 2009, 122, 248-256.e5. [CrossRef] [PubMed]

14. Bouter, K.E.; van Raalte, D.H.; Groen, A.K.; Nieuwdorp, M. Role of the Gut Microbiome in the Pathogenesis of Obesity and Obesity-Related Metabolic Dysfunction. Gastroenterology 2017, 152, 1671-1678. [CrossRef] [PubMed]

15. Singh, R.K.; Chang, H.-W.; Yan, D.; Lee, K.M.; Ucmak, D.; Wong, K.; Abrouk, M.; Farahnik, B.; Nakamura, M.; Zhu, T.H.; et al. Influence of diet on the gut microbiome and implications for human health. J. Transl. Med. 2017, 15, 73. [CrossRef] [PubMed]

16. Ridaura, V.K.; Faith, J.J.; Rey, F.E.; Cheng, J.; Duncan, A.E.; Kau, A.L.; Griffin, N.W.; Lombard, V.; Henrissat, B.; Bain, J.R.; et al. Gut microbiota from twins discordant for obesity modulate metabolism in mice. Science 2013, 341, 1241214. [CrossRef]

17. Alang, N.; Kelly, C.R. Weight gain after fecal microbiota transplantation. Open Forum Infect. Dis. 2015, 2, ofv004. [CrossRef] [PubMed]

18. Zhang, Z.; Mocanu, V.; Cai, C.; Dang, J.; Slater, L.; Deehan, E.C.; Walter, J.; Madsen, K.L. Impact of Fecal Microbiota Transplantation on Obesity and Metabolic Syndrome-A Systematic Review. Nutrients 2019, 11, 2291. [CrossRef] [PubMed]

19. Falony, G.; Joossens, M.; Vieira-Silva, S.; Wang, J.; Darzi, Y.; Faust, K.; Kurilshikov, A.; Bonder, M.-J.; Valles-Colomer, M.; Vandeputte, D.; et al. Population-level analysis of gut microbiome variation. Science 2016, 352, 560-564. [CrossRef] [PubMed] 
20. Zhernakova, A.; Kurilshikov, A.; Bonder, M.J.; Tigchelaar, E.F.; Schirmer, M.; Vatanen, T.; Mujagic, Z.; Vila, A.V.; Falony, G.; Vieira-Silva, S.; et al. Population-based metagenomics analysis reveals markers for gut microbiome composition and diversity. Science 2016, 352, 565-569. [CrossRef]

21. Kurilshikov, A.; van den Munckhof, I.C.L.; Chen, L.; Bonder, M.J.; Schraa, K.; Rutten, J.H.W.; Riksen, N.P.; de Graaf, J.; Oosting, M.; Sanna, S.; et al. Gut Microbial Associations to Plasma Metabolites Linked to Cardiovascular Phenotypes and Risk. Circ. Res. 2019, 124, 1808-1820. [CrossRef]

22. Martinez, K.B.; Pierre, J.F.; Chang, E.B. The Gut Microbiota: The Gateway to Improved Metabolism. Gastroenterol. Clin. North Am. 2016, 45, 601-614. [CrossRef] [PubMed]

23. Turnbaugh, P.J.; Bäckhed, F.; Fulton, L.; Gordon, J.I. Diet-induced obesity is linked to marked but reversible alterations in the mouse distal gut microbiome. Cell Host Microbe 2008, 3, 213-223. [CrossRef]

24. John, G.K.; Mullin, G.E. The Gut Microbiome and Obesity. Curr. Oncol. Rep. 2016, 18, 45. [CrossRef] [PubMed]

25. Gérard, P. Gut microbiota and obesity. Cell. Mol. Life Sci. 2016, 73, 147-162. [CrossRef] [PubMed]

26. Gils Contreras, A.; Bonada Sanjaume, A.; Montero Jaime, M.; Rabassa Soler, A.; Sabench Pereferrer, F.; Molina López, A.; Becerra Tomás, N.; Del Castillo Déjardin, D.; Salas-Salvadó, J. Effects of Two Preoperatory Weight Loss Diets on Hepatic Volume, Metabolic Parameters, and Surgical Complications in Morbid Obese Bariatric Surgery Candidates: A Randomized Clinical Trial. Obes. Surg. 2018, 28, 3756-3768. [CrossRef] [PubMed]

27. Gérard, P. Gut Microbiome and Obesity. How to Prove Causality? Ann. Am. Thorac. Soc. 2017, 14, S354-S356. [CrossRef]

28. Van Nieuwenhove, Y.; Dambrauskas, Z.; Campillo-Soto, A.; van Dielen, F.; Wiezer, R.; Janssen, I.; Kramer, M.; Thorell, A. Preoperative very low-calorie diet and operative outcome after laparoscopic gastric bypass: A randomized multicenter study. Arch. Surg. 2011, 146, 1300-1305. [CrossRef]

29. Sivakumar, J.; Chong, L.; Ward, S.; Sutherland, T.R.; Read, M.; Hii, M.W. Body Composition Changes Following a Very-Low-Calorie Pre-Operative Diet in Patients Undergoing Bariatric Surgery. Obes. Surg. 2020, 30, 119-126. [CrossRef]

30. Fried, M.; Yumuk, V.; Oppert, J.M.; Scopinaro, N.; Torres, A.; Weiner, R.; Yashkov, Y.; Frühbeck, G. Interdisciplinary European guidelines on metabolic and bariatric surgery. Obes. Surg. 2014, 24, 42-55. [CrossRef] [PubMed]

31. Welbourn, R.; Hollyman, M.; Kinsman, R.; Dixon, J.; Liem, R.; Ottosson, J.; Ramos, A.; Våge, V.; Al-Sabah, S.; Brown, W.; et al. Bariatric Surgery Worldwide: Baseline Demographic Description and One-Year Outcomes from the Fourth IFSO Global Registry Report 2018. Obes. Surg. 2019, 29, 782-795. [CrossRef]

32. Angrisani, L.; Santonicola, A.; Iovino, P.; Vitiello, A.; Higa, K.; Himpens, J.; Buchwald, H.; Scopinaro, N. IFSO Worldwide Survey 2016: Primary, Endoluminal, and Revisional Procedures. Obes. Surg. 2018, 28, 3783-3794. [CrossRef]

33. Toolabi, K.; Sarkardeh, M.; Vasigh, M.; Golzarand, M.; Vezvaei, P.; Kooshki, J. Comparison of Laparoscopic Roux-en-Y Gastric Bypass and Laparoscopic Sleeve Gastrectomy on Weight Loss, Weight Regain, and Remission of Comorbidities: A 5 Years of Follow-up Study. Obes. Surg. 2020, 30, 440-445. [CrossRef] [PubMed]

34. Sharples, A.J.; Mahawar, K. Systematic Review and Meta-Analysis of Randomised Controlled Trials Comparing Long-Term Outcomes of Roux-En-Y Gastric Bypass and Sleeve Gastrectomy. Obes. Surg. 2020, 30, 664-672. [CrossRef]

35. Raygor, V.; Garcia, L.; Maron, D.J.; Morton, J.M. The Comparative Effect of Roux-en-Y Gastric Bypass and Sleeve Gastrectomy on 10-Year and Lifetime Atherosclerotic Cardiovascular Disease Risk. Obes. Surg. 2019, 29, 3111-3117. [CrossRef] [PubMed]

36. Nguyen, N.T.; Varela, J.E. Bariatric surgery for obesity and metabolic disorders: State of the art. Nat. Rev. Gastroenterol. Hepatol. 2017, 14, 160-169. [CrossRef]

37. Baldwin, D.; Chennakesavalu, M.; Gangemi, A. Systematic review and meta-analysis of Roux-en-Y gastric bypass against laparoscopic sleeve gastrectomy for amelioration of NAFLD using four criteria. Surg. Obes. Relat. Dis. 2019, 15, 2123-2130. [CrossRef]

38. Fakhry, T.K.; Mhaskar, R.; Schwitalla, T.; Muradova, E.; Gonzalvo, J.P.; Murr, M.M. Bariatric surgery improves nonalcoholic fatty liver disease: A contemporary systematic review and meta-analysis. Surg. Obes. Relat. Dis. 2019, 15, 502-511. [CrossRef] [PubMed] 
39. Cherla, D.V.; Rodriguez, N.A.; Vangoitsenhoven, R.; Singh, T.; Mehta, N.; McCullough, A.J.; Brethauer, S.A.; Schauer, P.R.; Aminian, A. Impact of sleeve gastrectomy and Roux-en-Y gastric bypass on biopsy-proven non-alcoholic fatty liver disease. Surg. Endosc. 2020, 34, 2266-2272. [CrossRef]

40. Sridharan, K.; Kalayarasan, R.; Kamalanathan, S.; Sahoo, J.; Kar, S.S.; Nandhini, L.P.; Palui, R.; Durgia, H. Change in insulin resistance, beta cell function, glucagon-like peptide-1 and calcitonin levels two weeks after bariatric surgery. Diabetes Metab. Syndr. 2019, 13, 2142-2147. [CrossRef]

41. Carreau, A.-M.; Noll, C.; Blondin, D.P.; Frisch, F.; Nadeau, M.; Pelletier, M.; Phoenix, S.; Cunnane, S.C.; Guérin, B.; Turcotte, E.E.; et al. Bariatric Surgery Rapidly Decreases Cardiac Dietary Fatty Acid Partitioning and Hepatic Insulin Resistance Through Increased Intra-abdominal Adipose Tissue Storage and Reduced Spillover in Type 2 Diabetes. Diabetes 2020, 69, 567-577. [CrossRef]

42. Feng, W.; Yin, T.; Chu, X.; Shan, X.; Jiang, C.; Wang, Y.; Qian, Y.; Zhu, D.; Sun, X.; Bi, Y. Metabolic effects and safety of Roux-en-Y gastric bypass surgery vs. conventional medication in obese Chinese patients with type 2 diabetes. Diabetes. Metab. Res. Rev. 2019, 35, e3138. [CrossRef] [PubMed]

43. Kang, J.H.; Le, Q.A. Effectiveness of bariatric surgical procedures: A systematic review and network meta-analysis of randomized controlled trials. Medicine (Baltimore) 2017, 96, e8632. [CrossRef] [PubMed]

44. Gutiérrez-Repiso, C.; Moreno-Indias, I.; de Hollanda, A.; Martín-Núñez, G.M.; Vidal, J.; Tinahones, F.J. Gut microbiota specific signatures are related to the successful rate of bariatric surgery. Am. J. Transl. Res. 2019, 11, 942-952. [PubMed]

45. De Hollanda, A.; Ruiz, T.; Jiménez, A.; Flores, L.; Lacy, A.; Vidal, J. Patterns of Weight Loss Response Following Gastric Bypass and Sleeve Gastrectomy. Obes. Surg. 2015, 25, 1177-1183. [CrossRef] [PubMed]

46. Manning, S.; Pucci, A.; Carter, N.C.; Elkalaawy, M.; Querci, G.; Magno, S.; Tamberi, A.; Finer, N.; Fiennes, A.G.; Hashemi, M.; et al. Early postoperative weight loss predicts maximal weight loss after sleeve gastrectomy and Roux-en-Y gastric bypass. Surg. Endosc. 2015, 29, 1484-1491. [CrossRef] [PubMed]

47. Nicoletti, C.F.; de Oliveira, A.P.R.P.; Brochado, M.J.F.; Pinhel, M.A.S.; de Oliveira, B.A.P.; Marchini, J.S.; Dos Santos, J.E.; Salgado, W.; Cury, N.M.; de Araújo, L.F.; et al. The Ala55Val and -866GA polymorphisms of the UCP2 gene could be biomarkers for weight loss in patients who had Roux-en-Y gastric bypass. Nutrition 2017, 33, 326-330. [CrossRef]

48. Barhouch, A.S.; Padoin, A.V.; Casagrande, D.S.; Chatkin, R.; Süssenbach, S.P.; Pufal, M.A.; Rossoni, C.; Mottin, C.C. Predictors of Excess Weight Loss in Obese Patients After Gastric Bypass: A 60-Month Follow-up. Obes. Surg. 2016, 26, 1178-1185. [CrossRef]

49. Faria, G.; Preto, J.; Almeida, A.B.; Guimarães, J.T.; Calhau, C.; Taveira-Gomes, A. Fasting glycemia: A good predictor of weight loss after RYGB. Surg. Obes. Relat. Dis. 2014, 10, 419-424. [CrossRef]

50. Contreras, J.E.; Santander, C.; Court, I.; Bravo, J. Correlation between age and weight loss after bariatric surgery. Obes. Surg. 2013, 23, 1286-1289. [CrossRef]

51. Melton, G.B.; Steele, K.E.; Schweitzer, M.A.; Lidor, A.O.; Magnuson, T.H. Suboptimal weight loss after gastric bypass surgery: Correlation of demographics, comorbidities, and insurance status with outcomes. J. Gastrointest. Surg. 2008, 12, 250-255. [CrossRef]

52. Furet, J.-P.; Kong, L.-C.; Tap, J.; Poitou, C.; Basdevant, A.; Bouillot, J.-L.; Mariat, D.; Corthier, G.; Doré, J.; Henegar, C.; et al. Differential adaptation of human gut microbiota to bariatric surgery-induced weight loss: Links with metabolic and low-grade inflammation markers. Diabetes 2010, 59, 3049-3057. [CrossRef] [PubMed]

53. Fouladi, F.; Brooks, A.E.; Fodor, A.A.; Carroll, I.M.; Bulik-Sullivan, E.C.; Tsilimigras, M.C.B.; Sioda, M.; Steffen, K.J. The Role of the Gut Microbiota in Sustained Weight Loss Following Roux-en-Y Gastric Bypass Surgery. Obes. Surg. 2019, 29, 1259-1267. [CrossRef] [PubMed]

54. Ilhan, Z.E.; DiBaise, J.K.; Isern, N.G.; Hoyt, D.W.; Marcus, A.K.; Kang, D.-W.; Crowell, M.D.; Rittmann, B.E.; Krajmalnik-Brown, R. Distinctive microbiomes and metabolites linked with weight loss after gastric bypass, but not gastric banding. ISME J. 2017, 11, 2047-2058. [CrossRef] [PubMed]

55. Graessler, J.; Qin, Y.; Zhong, H.; Zhang, J.; Licinio, J.; Wong, M.-L.; Xu, A.; Chavakis, T.; Bornstein, A.B.; Ehrhart-Bornstein, M.; et al. Metagenomic sequencing of the human gut microbiome before and after bariatric surgery in obese patients with type 2 diabetes: Correlation with inflammatory and metabolic parameters. Pharm. J. 2013, 13, 514-522. [CrossRef] [PubMed] 
56. Tremaroli, V.; Karlsson, F.; Werling, M.; Ståhlman, M.; Kovatcheva-Datchary, P.; Olbers, T.; Fändriks, L.; Le Roux, C.W.; Nielsen, J.; Bäckhed, F. Roux-en-Y Gastric Bypass and Vertical Banded Gastroplasty Induce Long-Term Changes on the Human Gut Microbiome Contributing to Fat Mass Regulation. Cell Metab. 2015, 22, 228-238. [CrossRef]

57. Palleja, A.; Kashani, A.; Allin, K.H.; Nielsen, T.; Zhang, C.; Li, Y.; Brach, T.; Liang, S.; Feng, Q.; Jørgensen, N.B.; et al. Roux-en-Y gastric bypass surgery of morbidly obese patients induces swift and persistent changes of the individual gut microbiota. Genome Med. 2016, 8, 67. [CrossRef] [PubMed]

58. Patrone, V.; Vajana, E.; Minuti, A.; Callegari, M.L.; Federico, A.; Loguercio, C.; Dallio, M.; Tolone, S.; Docimo, L.; Morelli, L. Postoperative Changes in Fecal Bacterial Communities and Fermentation Products in Obese Patients Undergoing Bilio-Intestinal Bypass. Front. Microbiol. 2016, 7, 200. [CrossRef] [PubMed]

59. Murphy, R.; Tsai, P.; Jüllig, M.; Liu, A.; Plank, L.; Booth, M. Differential Changes in Gut Microbiota After Gastric Bypass and Sleeve Gastrectomy Bariatric Surgery Vary According to Diabetes Remission. Obes. Surg. 2017, 27, 917-925. [CrossRef]

60. Aron-Wisnewsky, J.; Prifti, E.; Belda, E.; Ichou, F.; Kayser, B.D.; Dao, M.C.; Verger, E.O.; Hedjazi, L.; Bouillot, J.-L.; Chevallier, J.-M.; et al. Major microbiota dysbiosis in severe obesity: Fate after bariatric surgery. Gut 2019, 68, 70-82. [CrossRef]

61. Paganelli, F.L.; Luyer, M.; Hazelbag, C.M.; Uh, H.-W.; Rogers, M.R.C.; Adriaans, D.; Berbers, R.-M.; Hendrickx, A.P.A.; Viveen, M.C.; Groot, J.A.; et al. Roux-Y Gastric Bypass and Sleeve Gastrectomy directly change gut microbiota composition independent of surgery type. Sci. Rep. 2019, 9, 10979. [CrossRef]

62. Liou, A.P.; Paziuk, M.; Luevano, J.-M.; Machineni, S.; Turnbaugh, P.J.; Kaplan, L.M. Conserved shifts in the gut microbiota due to gastric bypass reduce host weight and adiposity. Sci. Transl. Med. 2013, 5, 178 ra41. [CrossRef]

63. Sánchez-Alcoholado, L.; Gutiérrez-Repiso, C.; Gómez-Pérez, A.M.; García-Fuentes, E.; Tinahones, F.J.; Moreno-Indias, I. Gut microbiota adaptation after weight loss by Roux-en-Y gastric bypass or sleeve gastrectomy bariatric surgeries. Surg. Obes. Relat. Dis. 2019, 15, 1888-1895. [CrossRef] [PubMed]

64. Palmisano, S.; Campisciano, G.; Silvestri, M.; Guerra, M.; Giuricin, M.; Casagranda, B.; Comar, M.; de Manzini, N. Changes in Gut Microbiota Composition after Bariatric Surgery: A New Balance to Decode. J. Gastrointest. Surg. 2019. [CrossRef] [PubMed]

65. Kong, L.-C.; Tap, J.; Aron-Wisnewsky, J.; Pelloux, V.; Basdevant, A.; Bouillot, J.-L.; Zucker, J.-D.; Doré, J.; Clément, K. Gut microbiota after gastric bypass in human obesity: Increased richness and associations of bacterial genera with adipose tissue genes. Am. J. Clin. Nutr. 2013, 98, 16-24. [CrossRef] [PubMed]

66. Li, J.V.; Ashrafian, H.; Bueter, M.; Kinross, J.; Sands, C.; Le Roux, C.W.; Bloom, S.R.; Darzi, A.; Athanasiou, T.; Marchesi, J.R.; et al. Metabolic Surgery Profoundly Influences Gut Microbial-Host Metabolic Crosstalk. Gut 2011, 60, 1214-1223. [CrossRef] [PubMed]

67. Zhang, H.; DiBaise, J.K.; Zuccolo, A.; Kudrna, D.; Braidotti, M.; Yu, Y.; Parameswaran, P.; Crowell, M.D.; Wing, R.; Rittmann, B.E.; et al. Human gut microbiota in obesity and after gastric bypass. Proc. Natl. Acad. Sci. USA 2009, 106, 2365-2370. [CrossRef]

68. Kikuchi, R.; Irie, J.; Yamada-Goto, N.; Kikkawa, E.; Seki, Y.; Kasama, K.; Itoh, H. The Impact of Laparoscopic Sleeve Gastrectomy with Duodenojejunal Bypass on Intestinal Microbiota Differs from that of Laparoscopic Sleeve Gastrectomy in Japanese Patients with Obesity. Clin. Drug Investig. 2018, 38, 545-552. [CrossRef]

69. Wang, F.-G.; Bai, R.-X.; Yan, W.-M.; Yan, M.; Dong, L.-Y.; Song, M.-M. Differential composition of gut microbiota among healthy volunteers, morbidly obese patients and post-bariatric surgery patients. Exp. Ther. Med. 2019, 17, 2268-2278. [CrossRef]

70. Campisciano, G.; Cason, C.; Palmisano, S.; Giuricin, M.; Rizzardi, A.; Croce, L.S.; de Manzini, N.; Comar, M. Bariatric surgery drives major rearrangements of the intestinal microbiota including the biofilm composition. Front. Biosci. (Elite Ed) 2018, 10, 495-505.

71. Liu, R.; Hong, J.; Xu, X.; Feng, Q.; Zhang, D.; Gu, Y.; Shi, J.; Zhao, S.; Liu, W.; Wang, X.; et al. Gut microbiome and serum metabolome alterations in obesity and after weight-loss intervention. Nat. Med. 2017, 23, 859-868. [CrossRef]

72. Damms-Machado, A.; Mitra, S.; Schollenberger, A.E.; Kramer, K.M.; Meile, T.; Königsrainer, A.; Huson, D.H.; Bischoff, S.C. Effects of surgical and dietary weight loss therapy for obesity on gut microbiota composition and nutrient absorption. Biomed Res. Int. 2015, 2015, 806248. [CrossRef] [PubMed] 
73. Sanmiguel, C.P.; Jacobs, J.; Gupta, A.; Ju, T.; Stains, J.; Coveleskie, K.; Lagishetty, V.; Balioukova, A.; Chen, Y.; Dutson, E.; et al. Surgically Induced Changes in Gut Microbiome and Hedonic Eating as Related to Weight Loss: Preliminary Findings in Obese Women Undergoing Bariatric Surgery. Psychosom. Med. 2017, 79, 880-887. [CrossRef]

74. Ward, E.K.; Schuster, D.P.; Stowers, K.H.; Royse, A.K.; Ir, D.; Robertson, C.E.; Frank, D.N.; Austin, G.L. The effect of PPI use on human gut microbiota and weight loss in patients undergoing laparoscopic Roux-en-Y gastric bypass. Obes. Surg. 2014, 24, 1567-1571. [CrossRef] [PubMed]

75. Federico, A.; Dallio, M.; Tolone, S.; Gravina, A.G.; Patrone, V.; Romano, M.; Tuccillo, C.; Mozzillo, A.L.; Amoroso, V.; Misso, G.; et al. Gastrointestinal Hormones, Intestinal Microbiota and Metabolic Homeostasis in Obese Patients: Effect of Bariatric Surgery. In Vivo 2016, 30, 321-330. [PubMed]

76. Cortez, R.V.; Petry, T.; Caravatto, P.; Pessôa, R.; Sanabani, S.S.; Martinez, M.B.; Sarian, T.; Salles, J.E.; Cohen, R.; Taddei, C.R. Shifts in intestinal microbiota after duodenal exclusion favor glycemic control and weight loss: A randomized controlled trial. Surg. Obes. Relat. Dis. 2018, 14, 1748-1754. [CrossRef]

77. Pajecki, D.; de Oliveira, L.C.; Sabino, E.C.; de Souza-Basqueira, M.; Dantas, A.C.B.; Nunes, G.C.; de Cleva, R.; Santo, M.A. Changes in the intestinal microbiota of superobese patients after bariatric surgery. Clinics (Sao Paulo) 2019, 74, e1198. [CrossRef]

78. Lee, C.J.; Florea, L.; Sears, C.L.; Maruthur, N.; Potter, J.J.; Schweitzer, M.; Magnuson, T.; Clark, J.M. Changes in Gut Microbiome after Bariatric Surgery Versus Medical Weight Loss in a Pilot Randomized Trial. Obes. Surg. 2019, 29, 3239-3245. [CrossRef]

79. Shen, N.; Caixàs, A.; Ahlers, M.; Patel, K.; Gao, Z.; Dutia, R.; Blaser, M.J.; Clemente, J.C.; Laferrère, B. Longitudinal changes of microbiome composition and microbial metabolomics after surgical weight loss in individuals with obesity. Surg. Obes. Relat. Dis. 2019, 15, 1367-1373. [CrossRef]

80. Al Assal, K.; Prifti, E.; Belda, E.; Sala, P.; Clément, K.; Dao, M.-C.; Doré, J.; Levenez, F.; Taddei, C.R.; Fonseca, D.C.; et al. Gut Microbiota Profile of Obese Diabetic Women Submitted to Roux-en-Y Gastric Bypass and Its Association with Food Intake and Postoperative Diabetes Remission. Nutrients 2020, 12, 278. [CrossRef]

81. Janmohammadi, P.; Sajadi, F.; Alizadeh, S.; Daneshzad, E. Comparison of Energy and Food Intake Between Gastric Bypass and Sleeve Gastrectomy: A Meta-analysis and Systematic Review. Obes. Surg. 2019, 29, 1040-1048. [CrossRef] [PubMed]

82. Reichmann, M.T.F.; Todeschini, S.; Setter, N.; Vilela, R.M.; Radominski, R.B. Comparación de la ingesta dietética entre las mujeres en el postoperatorio tardío después del bypass gástrico en Y de Roux con la pirámide nutricional bariátrica. Nutr. Hosp. 2019, 36, 599-603. [CrossRef] [PubMed]

83. Soares, F.L.; Bissoni de Sousa, L.; Corradi-Perini, C.; Ramos da Cruz, M.R.; Nunes, M.G.J.; Branco-Filho, A.J. Food quality in the late postoperative period of bariatric surgery: An evaluation using the bariatric food pyramid. Obes. Surg. 2014, 24, 1481-1486. [CrossRef]

84. Guo, Y.; Liu, C.-Q.; Shan, C.-X.; Chen, Y.; Li, H.-H.; Huang, Z.-P.; Zou, D.-J. Gut microbiota after Roux-en-Y gastric bypass and sleeve gastrectomy in a diabetic rat model: Increased diversity and associations of discriminant genera with metabolic changes. Diabetes Metab. Res. Rev. 2017, 33. [CrossRef] [PubMed]

85. Haange, S.-B.; Jehmlich, N.; Krügel, U.; Hintschich, C.; Wehrmann, D.; Hankir, M.; Seyfried, F.; Froment, J.; Hübschmann, T.; Müller, S.; et al. Gastric bypass surgery in a rat model alters the community structure and functional composition of the intestinal microbiota independently of weight loss. Microbiome 2020, 8, 13. [CrossRef]

86. Flint, H.J.; Duncan, S.H.; Scott, K.P.; Louis, P. Links between diet, gut microbiota composition and gut metabolism. Proc. Nutr. Soc. 2015, 74, 13-22. [CrossRef] [PubMed]

87. Fava, F.; Gitau, R.; Griffin, B.A.; Gibson, G.R.; Tuohy, K.M.; Lovegrove, J.A. The type and quantity of dietary fat and carbohydrate alter faecal microbiome and short-chain fatty acid excretion in a metabolic syndrome 'at-risk' population. Int. J. Obes. (Lond) 2013, 37, 216-223. [CrossRef] [PubMed]

88. Wu, G.D.; Chen, J.; Hoffmann, C.; Bittinger, K.; Chen, Y.-Y.; Keilbaugh, S.A.; Bewtra, M.; Knights, D.; Walters, W.A.; Knight, R.; et al. Linking long-term dietary patterns with gut microbial enterotypes. Science 2011, 334, 105-108. [CrossRef] [PubMed]

89. David, L.A.; Maurice, C.F.; Carmody, R.N.; Gootenberg, D.B.; Button, J.E.; Wolfe, B.E.; Ling, A.V.; Devlin, A.S.; Varma, Y.; Fischbach, M.A.; et al. Diet rapidly and reproducibly alters the human gut microbiome. Nature 2014, 505, 559-563. [CrossRef] 
90. De Filippo, C.; Cavalieri, D.; Di Paola, M.; Ramazzotti, M.; Poullet, J.B.; Massart, S.; Collini, S.; Pieraccini, G.; Lionetti, P. Impact of diet in shaping gut microbiota revealed by a comparative study in children from Europe and rural Africa. Proc. Natl. Acad. Sci. USA 2010, 107, 14691-14696. [CrossRef]

91. Mills, S.; Stanton, C.; Lane, J.A.; Smith, G.J.; Ross, R.P. Precision Nutrition and the Microbiome, Part I: Current State of the Science. Nutrients 2019, 11, 923. [CrossRef]

92. Ravussin, Y.; Koren, O.; Spor, A.; LeDuc, C.; Gutman, R.; Stombaugh, J.; Knight, R.; Ley, R.E.; Leibel, R.L. Responses of gut microbiota to diet composition and weight loss in lean and obese mice. Obesity (Silver Spring) 2012, 20, 738-747. [CrossRef] [PubMed]

93. Louis, S.; Tappu, R.-M.; Damms-Machado, A.; Huson, D.H.; Bischoff, S.C. Characterization of the Gut Microbial Community of Obese Patients Following a Weight-Loss Intervention Using Whole Metagenome Shotgun Sequencing. PLoS ONE 2016, 11, e0149564. [CrossRef]

94. Heinsen, F.-A.; Fangmann, D.; Müller, N.; Schulte, D.M.; Rühlemann, M.C.; Türk, K.; Settgast, U.; Lieb, W.; Baines, J.F.; Schreiber, S.; et al. Beneficial Effects of a Dietary Weight Loss Intervention on Human Gut Microbiome Diversity and Metabolism Are Not Sustained during Weight Maintenance. Obes. Facts 2016, 9 , 379-391. [CrossRef] [PubMed]

95. Ott, B.; Skurk, T.; Hastreiter, L.; Lagkouvardos, I.; Fischer, S.; Büttner, J.; Kellerer, T.; Clavel, T.; Rychlik, M.; Haller, D.; et al. Effect of caloric restriction on gut permeability, inflammation markers, and fecal microbiota in obese women. Sci. Rep. 2017, 7, 11955. [CrossRef]

96. Lewis, M.C.; Phillips, M.L.; Slavotinek, J.P.; Kow, L.; Thompson, C.H.; Toouli, J. Change in liver size and fat content after treatment with Optifast very low calorie diet. Obes. Surg. 2006, 16, 697-701. [CrossRef] [PubMed]

97. Chakravartty, S.; Vivian, G.; Mullholland, N.; Shaikh, H.; McGrath, J.; Sidhu, P.S.; Jaffer, O.; Patel, A.G. Preoperative liver shrinking diet for bariatric surgery may impact wound healing: A randomized controlled trial. Surg. Obes. Relat. Dis. 2019, 15, 117-125. [CrossRef] [PubMed]

(C) 2020 by the authors. Licensee MDPI, Basel, Switzerland. This article is an open access article distributed under the terms and conditions of the Creative Commons Attribution (CC BY) license (http://creativecommons.org/licenses/by/4.0/). 